ARTICLE

Received 10 May 2014 | Accepted 12 Jan 2015 | Published 19 Feb $2015 \quad$ DOl: 10.1038/ncomms7286

\title{
Generation of a ciliary margin-like stem cell niche from self-organizing human retinal tissue
}

\author{
Atsushi Kuwahara ${ }^{1,2,3, \star, \dagger}$, Chikafumi Ozone ${ }^{1,4, \star}$, Tokushige Nakano $1,2,3, \star$, Koichi Saito $^{3}$, Mototsugu Eiraku ${ }^{5} \&$ \\ Yoshiki Sasai ${ }^{1,2, \ddagger}$
}

In the developing neural retina (NR), multipotent stem cells within the ciliary margin (CM) contribute to de novo retinal tissue growth. We recently reported the ability of human embryonic stem cells (hESCs) to self-organize stratified NR using a three-dimensional culture technique. Here we report the emergence of $\mathrm{CM}$-like stem cell niches within human retinal tissue. First, we developed a culture method for selective NR differentiation by timed BMP4 treatment. We then found that inhibiting GSK3 and FGFR induced the transition from NR tissue to retinal pigment epithelium (RPE), and that removing this inhibition facilitated the reversion of this RPE-like tissue back to the NR fate. This step-wise induction-reversal method generated tissue aggregates with RPE at the margin of central-peripherally polarized NR. We demonstrate that the NR-RPE boundary tissue further self-organizes a niche for CM stem cells that functions to expand the NR peripherally by de novo progenitor generation.

\footnotetext{
${ }^{1}$ Neurogenesis and Organogenesis Group, RIKEN Center for Developmental Biology, 2-2-3 Manatojima-Minamimachi, Chuo, Kobe 650-0047, Japan.

${ }^{2}$ Human Stem Cell Technology Unit, RIKEN Center for Developmental Biology, 2-2-3 Minatojima-minamimachi, Chuo, Kobe 650-0047, Japan.

${ }^{3}$ Environmental Health Science Laboratory, Sumitomo Chemical Co., Ltd., 3-1-98 Kasugade-naka, Konohana, Osaka 554-8558, Japan. ${ }^{4}$ Department of Endocrinology and Diabetes, Graduate School of Medicine, Nagoya University, 65 Tsurumai-cho, Showa, Nagoya 466-8550, Japan. ${ }^{5}$ Four-Dimensional Tissue Analysis Unit, RIKEN Center for Developmental Biology, 2-2-3 Minatojima-minamimachi, Chuo, Kobe 650-0047, Japan. * These authors contributed equally to this work. †Present address: Regenerative \& Cellular Medicine Office, Sumitomo Dainippon Pharma Co., Ltd., 2-2-2, Minatojima-minamimachi, Chuo, Kobe 650-0047, Japan. Correspondence and requests for materials should be addressed to A.K. (email: kuwaharaa@cdb.riken.jp) and M.E. (email: eiraku@cdb.riken.jp).

声Deceased.
} 
T he retina is the main visual sensory tissue in mammals. Its anlage, the optic cup, is derived from the rostral diencephalon and is composed of the inner and outer walls-the neural retina (NR) and retinal pigment epithelium (RPE), respectively ${ }^{1,2}$. Mammalian NR is known to possess a low regenerative capacity, and spontaneous tissue recovery hardly occurs following substantial damage caused by trauma or degenerative diseases. This stands in sharp contrast to the newt NR, for instance, which can fully regenerate from neighbouring RPE even after total resection (reviewed in refs 3,4).

In vertebrate retinogenesis, the NR has two different groups of progenitors present in distinct locations. During early retinal development, epithelial progenitors are widely present in the NR epithelium and function to generate photoreceptors and other types of retinal neurons ${ }^{5}$. Their differentiation capacity gradually changes as retinogenesis proceeds; they have a tendency to produce ganglion cells and cones at early stages, and then rods and bipolar cells at late stages, ${ }^{6,7}$. Like cortical progenitors, these NR progenitors show the radial glia-like morphology with thin apical and basal processes, characteristic of neuroepithelial progenitors, and they undergo interkinetic nuclear migration during mitotic cycles ${ }^{8,9}$. (Hereafter, these progenitors are referred to as 'NR progenitors').

Another kind of progenitor is located at the peripheral margin of the NR, the ciliary margin $(\mathrm{CM})^{5,10,11}$. The teleost (fish) eye contains an active stem cell system in the CM zone (also called circumferential germinal zone) that contributes to NR expansion by de novo generation of the tissue even in adulthood ${ }^{12}$. This type of stem cell robustly self-renews and gives rise to all NR cell types in the extending NR periphery, unlike the nonmarginal-NR progenitor, whose developmental competence gradually becomes confined to the generation of limited cell types during retinogenesis as mentioned above. In the chick embryo, the stem cell system at the CM also plays an active role during eye development, and stem cells isolated from this region can form NR tissue in floating aggregate culture ${ }^{13}$. The mammalian foetal eye also contains a peripheral NR zone resembling the chick embryonic CM in molecular marker expression, and this zone contains a substantial number of stem cells ${ }^{10,14,15}$ (Hereafter, these cells are referred to as ' $\mathrm{CM}$ stem cells'). The presence of a small number of retinal stem cells at the ciliary region in the adult mammalian eye has also been reported ${ }^{16-20}$, although the extent of their in vivo contribution to the maintenance and regeneration of adult retinal tissue remains elusive.

Recent advancement in stem cell research has enabled in vitro differentiation of retinal progenitors and their derivatives from pluripotent stem cells ${ }^{21-29}$. Moreover, mouse and human embryonic stem cells (hESC) aggregate have been shown to possess the potential to self-form optic cups in three-dimensional (3D) culture when retinal differentiation efficiency is sufficiently high $^{30-32}$. Notably, the ESC-derived NR self-organizes the formation of multiple retinal layers reminiscent of the postnatal retina. NR progenitors in this culture exhibit radial glia-like epithelial morphology, frequently proliferate with interkinetic nuclear migration and give birth to photoreceptors and other retinal neurons in a stage-dependent manner ${ }^{30,32}$, a process which largely recapitulates the activity of NR progenitors in vivo. Moreover, a recent report using mouse ES cells showed that self-forming NR tissue can give rise to functional photoreceptors capable of light response after grafting in the murine eye ${ }^{33}$.

In the self-organized optic cup, the boundary domain between NR and RPE, called the hinge domain, contains cells with a morphology distinct from that of NR and RPE cells. However, whether CM-type stem cells are generated at this region in longterm culture has remained unexplored. In the present study, we have uncovered the formation of CM-like zones in self-organizing retinal culture. First, we made substantial improvements to the 3D culture method of hESCs for promoting retinal differentiation. As reported previously, the formation of optic cup in hESC culture is less frequent $(\sim 10 \% \text { of aggregates })^{32}$ than in mouse ESC culture. Its efficacy is also influenced by minute changes in culture conditions. In particular, our previous culture included Matrigel, a crude extract of extracellular matrix from mouse EHS sarcoma cells, for promotion of retinal differentiation and epithelialization $^{32}$. The use of Matrigel is problematic because this undefined extract shows substantial batch-to-batch differences in promoting retinal differentiation. Furthermore, the use of animal-derived matrix proteins is also unfavourable from the viewpoint of future clinical applications.

Therefore, we established culture conditions for inducing selective retinal differentiation without the use of Matrigel. Using this new 3D culture technique, we further developed a two-step method that allows a reproducible formation of NR-RPE junctions to generate CM-like zones. Combining these new methods, we demonstrate that CM-like zones self-organize in vitro and function as growth zones in the periphery of hESC-derived NR.

\section{Results}

Highly selective self-formation of NR with BMP treatment. We observed in a separate study of cortical self-organization that hESC aggregates selectively differentiated into Foxg ${ }^{+}$telencephalic neuroepithelium when cultured in growth factor-free chemically defined medium (gfCDM) without Matrigel (Fig. 1a). While retinal differentiation was rarely seen under these conditions, we reasoned that the addition of certain signalling molecules to the medium might cause a shift of positional information from the telencephalic to the retinal direction, given the proximity of their anlages in the embryonic brain.

We therefore examined the effects of several candidate signals that are known to affect retinal differentiation (see Methods for details). Using Venus-knock-in reporter hESCs for $R x$ (retinal marker) ${ }^{32,34}$ expression, we observed that a transient treatment of hESC aggregates with a low concentration of Bone morphogenetic protein-4 (BMP4; starting at $1.5 \mathrm{nM}$ on day 6 and subject to half-dilution every 3 rd day) dramatically increased $R x::$ Venus expression (Fig. 1b-d; $>95 \%$ of hESC aggregates contained Venus $^{+}$epithelium on day 24; the timed application is important, since BMP4 treatment from day 0 did not promote retinal nor neural differentiation). Most of the BMP4-induced Venus $^{+}$epithelium was positive for Rx, Chx10 (ref. 35), Pax6 and Sox2 (neural retinal marker), and negative for Foxg1 and the RPE marker Mitf (Fig. 1e-i and Supplementary Fig. 1a), reminiscent of the expression profile of NR epithelium. This NR epithelium reproducibly contained phospho-Histone $\mathrm{H}^{+}$ mitotic cells on the outer surface, which accumulated the apical marker aPKC (Fig. 1j,k; also showing strong N-cadherin localization there). The basement membrane positive for Laminin and Collagen IV (Fig. 11 and Supplementary Fig. 1b) spontaneously formed on the luminal side in this culture without the addition of exogenous matrix proteins such as Matrigel. The induction efficacy of $R x:$ :AcGFP (a separately derived reporter line) exceeded $80 \%$ at the cellular level (Fig. $1 \mathrm{~m}-\mathrm{p}$ and Supplementary Fig. 1c,d), which is substantially higher than the efficiency seen in previous Matrigel-based cultures ${ }^{32}$.

Thus, our new culture method enabled efficient generation of human NR epithelium under nearly defined conditions except for the inclusion of a commonly used knockout serum replacement (KSR) additive. On day 50, the epithelia were continuous 

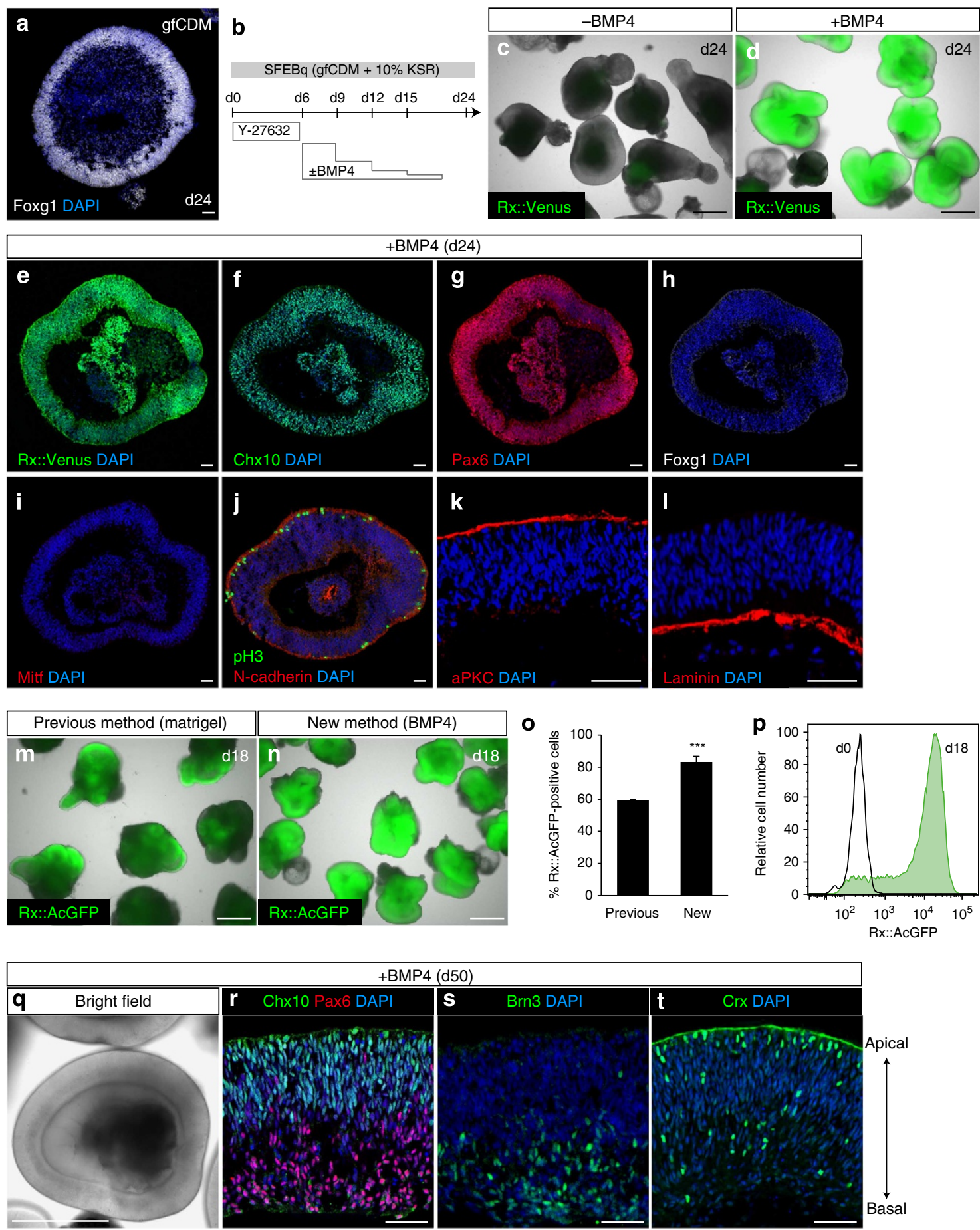

Figure 1 | Selective NR generation from hESC without using Matrigel. (a) Selective generation of Foxg1 ${ }^{+}$telencephalic neuroectoderm (day 24$)$ in 3D hESC culture using gfCDM without Matrigel and BMP4. Foxg1 (white) and nuclear staining with DAPI (blue). (b) Timing of BMP4 treatment. BMP4 $(1.5 \mathrm{nM})$ was added to medium on day 6 , while its concentration was diluted into half by half medium change on days 9,12 and 15 . (c, d) Induction of Rx::Venus by transient BMP4 treatment (d;c, untreated control) in hESC aggregates. (e-I) Immunostaining of NR tissue (day 24) generated by the improved culture method with antibodies for Venus (e), Chx10 (f), Pax6 (g), Foxg1 (h; no substantial expression), Mitf (i; no substantial expression), pH3 (j; green), $\mathrm{N}$-cadherin (j; red), aPKC ( $\mathbf{k}$; note the strong expression on the surface of spheroid) and Laminin (I). Blue, nuclear staining with DAPI.

$(\mathbf{m}-\mathbf{p})$ Comparison of Rx::AcGFP-inducing efficiency between our previous (m) and improved (new) (n) methods. (o) Percentage of AcGFP + cells in each culture on day 18 analyzed by FACS. Data represent mean \pm s.e.m. of three independent experiments (each experiment with 24 aggregates). ${ }^{\star \star \star} P<0.001$. Student's $t$-test. Representative FACS patterns for day 18 culture by the present method (green) and control undifferentiated Rx::AcGFP hESCs (day 0; black) are shown in $\mathbf{p}$. (q-t) Stratified NR tissue generated by the improved method. (q) Bright field view showing the formation of continuously thick NR (day 50). ( $\mathbf{r}-\mathbf{t})$ Pax6 ( $\mathbf{r}$; red) and Brn3 (s) were expressed in the cell layer on the basal side (ganglion cells), while Chx10 ${ }^{+}$progenitors ( $\mathbf{r}$; green) were located in the apical layer. NR at this stage contained moderate numbers of $\mathrm{Crx}^{+}$photoreceptor precursors (t) both on the apical and basal sides. Scale bars, $500 \mu \mathrm{m}(\mathbf{c}, \mathbf{d}, \mathbf{m}, \mathbf{n}, \mathbf{q})$ and $50 \mu \mathrm{m}(\mathbf{a}, \mathbf{e}-\mathbf{l}, \mathbf{r}-\mathbf{t})$. See also Supplementary Fig. 1. 
and thick (Fig. 1q), and contained cells expressing NR cell typespecific markers such as Brn3 and Pax6 for ganglion cells (Fig. 1r,s), Crx for photoreceptors (Fig. 1t), Calretinin for ganglion cells and interneurons (Supplementary Fig. 1e), and Ptfla for interneurons (Supplementary Fig. 1f) in addition to Chx10 ${ }^{+}$NR progenitors (Fig. 1r).

Taken together, these observations show that selective NR differentiation from hESCs without the use of Matrigel is enabled simply by a transient early BMP4 treatment. The strong promotion of NR differentiation by BMP4 treatment was rather unexpected, since the inductive role of BMP signals is known for RPE specification ${ }^{36}$ but not for early NR differentiation. The BMP4 treatment was transiently given at the early phase of culture, while the NR-RPE specification occurs later around day 18-20 and optic cup formation around day 24 (ref. 32). Therefore, we speculate that this effect could be relevant to the role of BMP signals in early forebrain patterning (this point is discussed later).

Generation of RPE next to NR by induction-reversal culture. In the embryonic eye, the $\mathrm{CM}$ arises from the hinge region joining the NR and RPE. Although the BMP-induced retinal differentiation culture was highly efficient, the retinal epithelium mostly consisted of NR (Fig. 1f), lacking RPE or a NR-RPE junction (Fig. 1i). This is consistent with the idea that NR differentiation is the default direction of retinal differentiation in ESC culture ${ }^{30}$. Our previous hESC study ${ }^{32}$ showed that RPE differentiation could be induced from hESC-derived retinal epithelium by treating with canonical Wnt signals from day 18 (RPE-inducing roles of Wnt signals in vivo are also reported and discussed in refs 37-40). Conversely, FGF signals have been shown to promote NR differentiation and suppress RPE differentiation ${ }^{41,42}$. Based on these effects (summarized in Supplementary Fig. 2), we next sought to induce RPE differentiation in a limited domain within the hESC-derived retinal epithelium.

When BMP-induced $\mathrm{Chx} 10^{+}$retinal epithelium was treated with the GSK3 inhibitor CHIR99021 (Wnt agonist) and the FGFR inhibitor SU5402 for 6 days from day 18 (Fig. 2a, condition 2), the epithelium became thin and expressed Mitf but not Chx10 on day 24 (these RPE features were found in $>90 \%$ of aggregates), whereas most of the nontreated epithelium (Fig. 2a, condition 1) remained thick and $\mathrm{Chx} 10^{+} / \mathrm{Mitf}^{-}$(Fig. 2b). Rx::Venus expression, which was initially strong on day 18 , became dim in the epithelium after treatment with GSK3 and FGFR inhibitors (see below; Rx expression is much weaker in RPE than in NR). Thus, Wnt signal augmentation and FGF signal inhibition for a relatively short period was sufficient to induce RPE-type epithelium from $\mathrm{Chx} 10^{+} \mathrm{NR}$ progenitors.

Interestingly, the fate of the induced $\mathrm{Mitf}^{+}$epithelial cells was not irreversibly fixed even after they exhibited these molecular and morphological characteristics of RPE (Fig. 2b). When hESCderived retinal epithelium was treated with CHIR99021 and SU5402 (days 18-24) and subsequently cultured in NRdifferentiation medium (not containing these inhibitors) from day 24 (Fig. 2a, condition 3), the majority of epithelium (in $\sim 70 \%$ of aggregates) repressed Mitf expression in their major portions, regained Chx10 expression and increased the epithelial thickness (Fig. 2b-e). This stands in contrast to the thin Mitf1 ${ }^{+}$ epithelium cultured continuously in the presence of the CHIR99021 (condition 2; Fig. 2a,b). Live imaging analysis (Supplementary Movies 1 and 2, Fig. 2f-i) showed that the recovery of the $R x::$ Venus-strong thick epithelium (NR type) in the aggregates did not occur by rapid local regrowth of residual NR-type epithelium but by the transition from $R x:$ Venus-dim thin epithelium (RPE type) to $R x::$ Venus-strong thick epithelium. In fact, recovery of epithelial thickness and $R x::$ Venus levels was already seen within the first few days after removal of GSK3 and FGFR inhibitors (Fig. 2f-i). This suggests that retinal differentiation, even after the appearance of early RPE features, can be reversed and redirected towards the NR fate simply by culturing the tissue in NR-differentiation medium. Hereafter, we refer to this step-wise culture (RPE induction followed by reversal to NR epithelium) as 'induction-reversal' culture (its possible mechanism is interpreted later in Discussion).

On day 35, in contrast to NR- and RPE-inducing conditions 1 and 2 (Fig. 3a,b), $\sim 40 \%$ of retinal aggregates in the inductionreversal culture (condition 3) exhibited a characteristic twodomain pattern: a large semispherical domain of continuous NR epithelium (with strong $R x:$ :Venus expression) with a small pigmented domain (or a few small domains) of thin and winding RPE in an adjacent location (Fig. 3c). This type of aggregates (two-domain aggregates, hereafter) could be easily selected (even without using microscope) for further culture (Fig. 3d at day 60). Figure $3 \mathrm{e}$ shows quantitative comparison of two-domain aggregate generation (shown in orange) between conditions 1, 2 and 3 at day 35 .

Immunostaining on day 50 showed that the two-domain aggregate contained continuous NR epithelium positive for $R x::$ Venus and Chx10 (Fig. 3f). Notably, the NR epithelium exhibited a gradual change in shape along the proximal-distal axis relative to RPE; the closer to RPE, the thinner the NR epithelium. In addition, molecular marker distribution exhibited an intriguing graded pattern. During in vivo development, differentiation of fate-specified retinal cells starts earlier in the central domain and is delayed in the peripheral portion ${ }^{43,44}$. For instance, Pax6 is initially expressed at high levels in all $\mathrm{Chx}_{10}{ }^{+} \mathrm{NR}$ progenitors. However, as neurogenesis begins in the NR, Pax6 expression becomes attenuated in progenitors except in the peripheral domain, and becomes strong in postmitotic ganglion cells $\left(\mathrm{Pax}^{+} / \mathrm{Chx} 0^{-}\right)$in the central domain. In hESC-derived retina, strong expression of Pax6 in $\mathrm{Ch \times 10}{ }^{+}$progenitors was seen preferentially in the NR margin next to the NR-RPE junction (Fig. 3f,g; arrow, junction; bracket, NR margin), whereas Pax6 ${ }^{+} / \mathrm{Chx}^{-} 0^{-}$ganglion cells were mostly found in the nonmarginal portion of the NR (Fig. 3g, arrowheads). Consistent with this idea, the nonmarginal NR portion also contained more cells positive for the postmitotic neuronal markers Brn3, TuJ1 and Calretinin (Fig. 3h-j) than the marginal NR portion. Moreover, differentiated photoreceptors marked by Blimp1, Recoverin or NRL (Fig. 3k-n; Supplementary Fig. 3a,b, see Methods) were preferentially located in the NR epithelium at a distance from the NR-RPE junction, indicating that differentiation of NR is more advanced in the nonmarginal NR portion (hereafter, the 'marginal'-'nonmarginal' axis is referred to as the peripheral-central axis, in analogy to the in vivo situation).

Collectively, these observations demonstrate that our 3D hESC culture, in which selective retinal differentiation and the induction-reversal process are combined, enables the adjacent formation of NR and RPE tissues. In the two-domain retinal structure generated by this culture, the continuity and integrity of NR epithelium was more efficiently maintained in long-term culture. While the aggregates carrying only NR (those generated typically in condition 1) tended to be discontinuous and break into small rosettes in culture beyond day 60 (Supplementary Fig. 3c, left), the NR in the two-domain aggregates (condition 3) more frequently contained continuous epithelial structures (Supplementary Fig. 3c,d).

The continuous NR exhibited a graded regional difference (or polarity) along the central-peripheral axis. In addition, this NR 
a

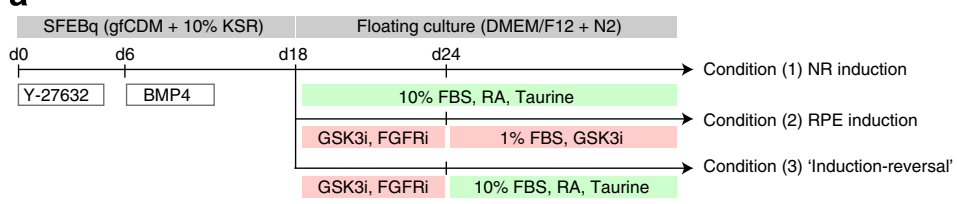

b

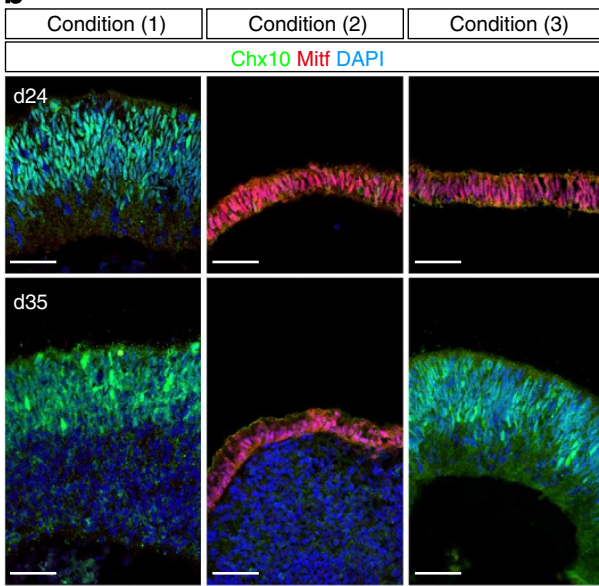

d

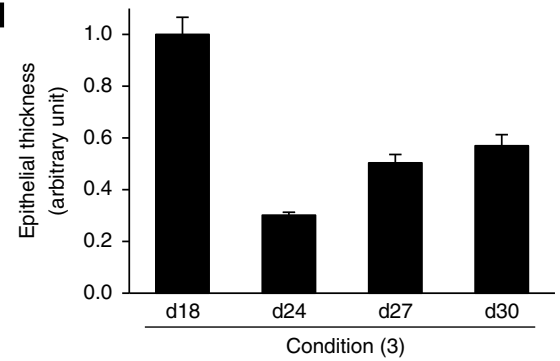

f

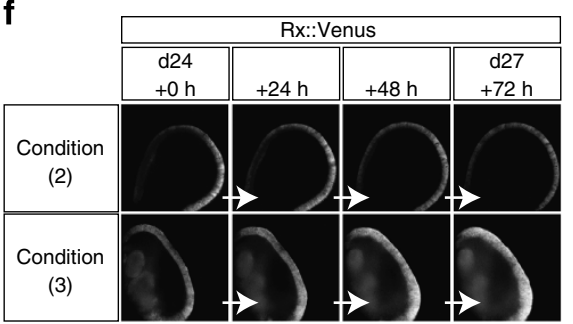

i
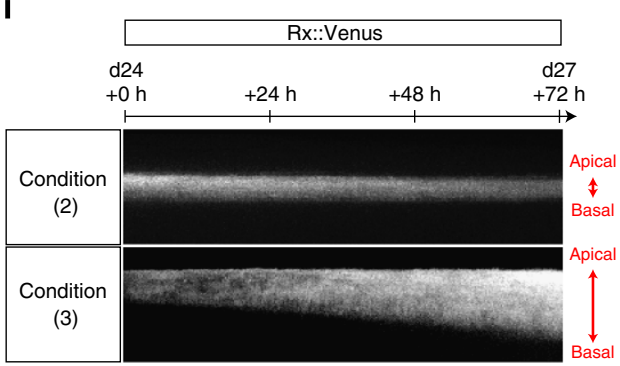

c

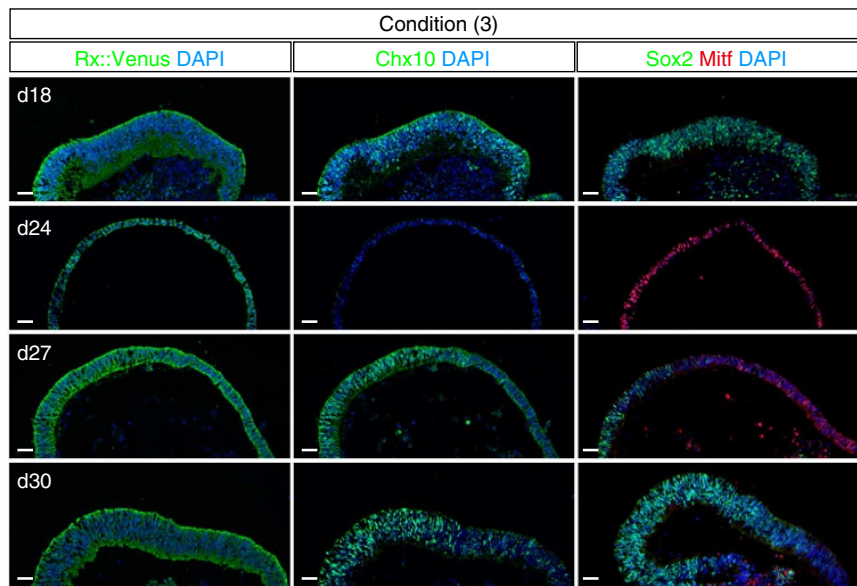

e

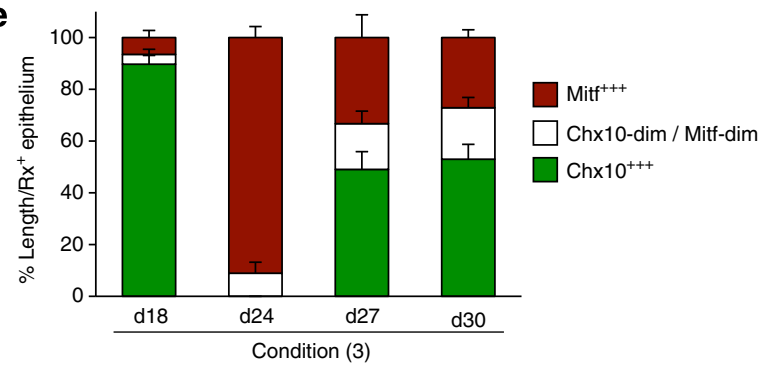

g

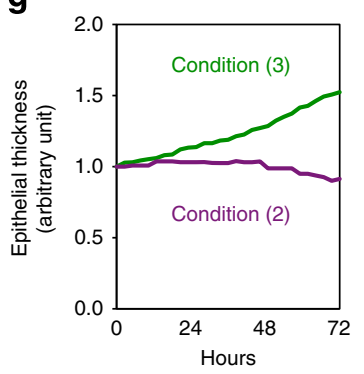

h

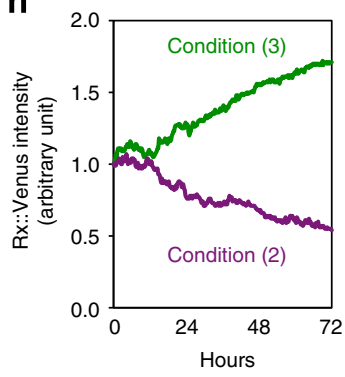

Figure 2 | NR-RPE conversion in induction-reversal culture. (a) Time table for three culture conditions. (b) Immunostaining of retinal epithelia cultured under conditions 1 (left), 2 (middle) and 3 (right) on day 24 (top) and day 35 (bottom). Under condition 2 on day 35, debris and mesenchymal cells were located basally under Mitf ${ }^{+}$epithelia. (c-e) Retinal epithelia cultured under condition 3 on day 18, 24, 27 and 30. (c) Immunostaining. (d) Change in epithelial thickness (fold change relative to day 18) during induction-reversal culture. Data represent mean \pm s.e.m. (each experiment with three representative points per aggregates (eight aggregates); each point was at the middle area of epithelial tissue). (e) Change in percentages of the length of the apical surface of Chx10-strong (green), Chx10-dim/Mitf-dim (white) or Mitf-strong (brown) epithelium in the Rx::Venus ${ }^{+}$epithelium during inductionreversal culture. Data represent mean \pm s.e.m. (each experiment with eight aggregates). (f-i) Live-imaging analysis (f) of changes in epithelial thickness ( $g$; fold change relative to $\mathrm{O} h$ ) and $R x:: V e n u s$ signal intensity ( $h$; fold change relative to $0 h$; mean $R x:: V e n u s$ intensity in NR epithelium was shown) during induction-reversal culture. 0 h, day 24. conditions 2 and 3, top and bottom (f) or purple and green lines $(\mathbf{g}, \mathbf{h})$, respectively. Note that the increase of epithelial thickness and Rx::Venus signal intensity cultured under condition 3. (i) Kymograph of live imaging data of Rx::Venus ${ }^{+}$retinal epithelia cultured under conditions 2 (top) and 3 (bottom) from day 24 to day 27. Red arrows represent apico-basal polarity. Scale bars, $50 \mu \mathrm{m}$ (b,c). See also Supplementary Fig. 2. 

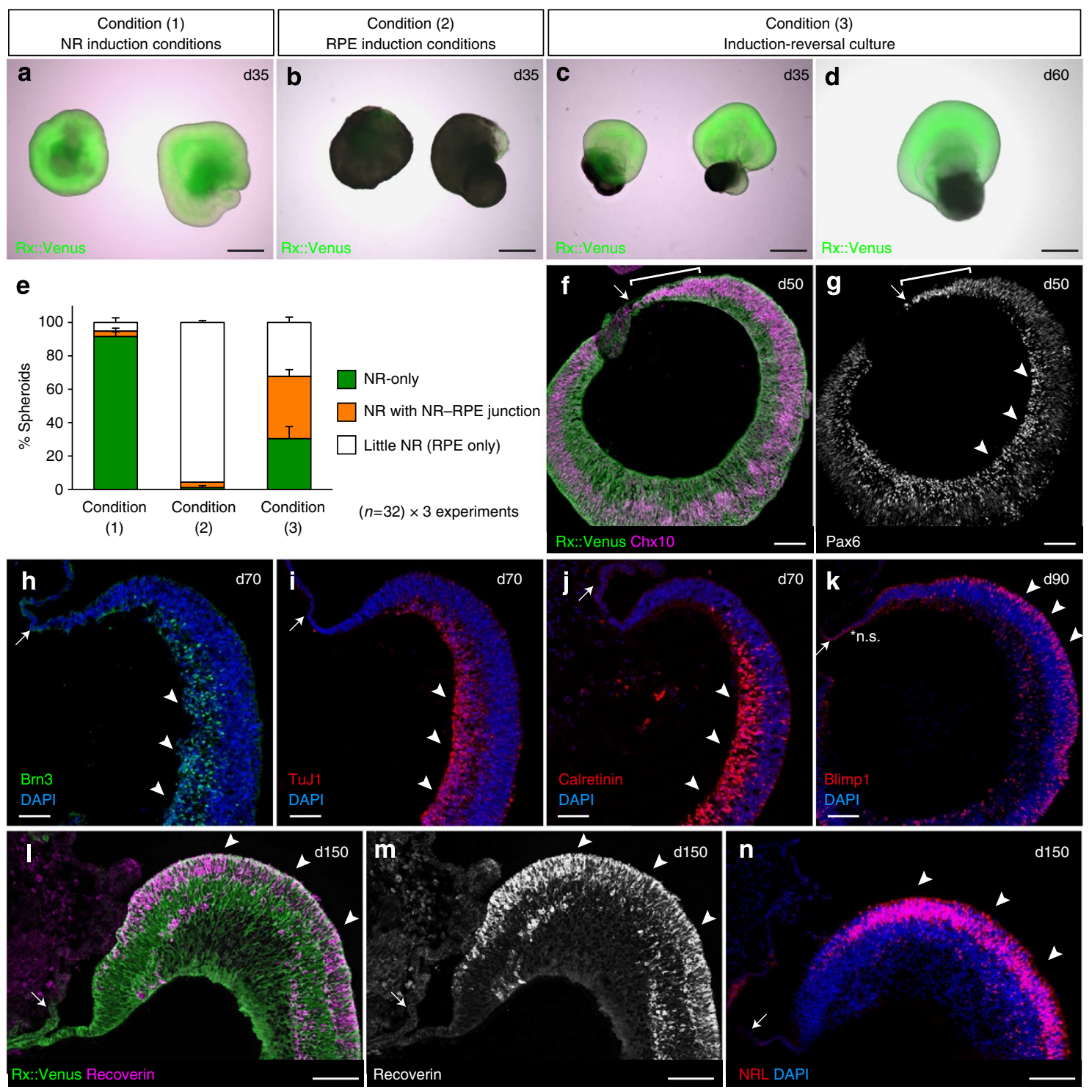

Figure 3 | Co-generation of NR and RPE by induction-reversal culture. (a-e) External appearance of self-formed NR and RPE structures under different conditions on day $35(\mathbf{a}-\mathbf{c}, \mathbf{e})$ and day 60 (d). (a) condition 1, (b) condition 2, (c,d) condition 3. (e) Percentages of three types of aggregates. Orange corresponds to the two-domain aggregates seen in c. Data represent mean \pm s.e.m. ( $n=3$ experiments, each experiment with 32 aggregates). (f-n) Immunostaining of NR tissues in the two-domain aggregates sectioned along the central-peripheral axis. (f,g) Day 50. (h-j) day 70. (k) day 90. (I-n) day 150. Arrows indicate the NR-RPE junctions $(\mathbf{f}-\mathbf{n})$. Arrowheads and brackets $(\mathbf{f}, \mathbf{g})$, the central portion abundant with Pax6 ${ }^{+} / \mathrm{Ch} 10^{-}$cells (arrowheads) and the peripheral portion preferentially containing Pax6 ${ }^{+} / \mathrm{Chx} 10^{+}$cells (bracket). Arrowheads (h-n), the central portion abundant with Brn3 (h), TuJ1 (i), Calretinin (j), Blimp1 (k), Recoverin (I,m) and NRL (n). ${ }^{\star}$ n.s., nonspecific staining. Scale bars, $500 \mu \mathrm{m}(\mathbf{a}-\mathbf{d})$ and $100 \mu \mathrm{m}(\mathbf{f}-\mathbf{n})$. See also Supplementary Fig. 3.

margin that was adjacent to RPE had a characteristic 'tapering' morphology of epithelium, which is also seen in the periphery of the foetal $\mathrm{NR}^{10,45}$. Therefore, we became intrigued by the possibility that this NR-RPE junction may have some similarity to the embryonic $\mathrm{CM}$, and thus examined this region for the expression of CM-characteristic molecular markers.

Expression of CM markers in the self-organized retina. In hESC-derived retina (Fig. 4a-h; day 63), the NR-RPE junctional region expressed a set of regional markers characteristic for the embryonic CM in a complex but spatially controlled manner (refs 45-49 and Supplementary Fig. 4a). The thin $\mathrm{Mitf}^{+}$epithelium (Fig. 4a) near the junction expressed the ciliary epithelium marker Aquaporin1 (Aqp1; Fig. 4b) and the
CM marker Otx1 (Fig. 4c). In contrast, the CM markers Zic1, Rdh10 and SSEA1 (Fig. 4d,f,g and Supplementary Fig. 4b-d), as well as the NR marker Sox2 (Fig. 4f) were expressed only in the Chx $10^{+}$domain (Fig. 4e). Interestingly, Aqp1 and Otx1 were expressed across the junction, that is, in both $\mathrm{Mitf}^{+}$and $\mathrm{Chx} 10^{+}$ epithelia (Fig. 4b,c).

The tapered NR epithelium, showing a gradual increase in thickness going distally from the thin $\mathrm{Mitf}^{+}$epithelium, appeared to have two subdomains. The $\mathrm{Chx} 10^{+} \mathrm{NR}$ margin directly next to the thin $\mathrm{Mitf}^{+}$epithelium (hereafter, subdomain 1 (sd 1)) expressed CM markers such as Otx1, SSEA1, Zic1 and Rdh10, but not fate-specific NR cell markers such as TuJ1 and Crx::Venus (Fig. 4c-h and Supplementary Fig. 4b-d; brackets). In contrast, the region of the tapering NR epithelium that lies distally to sd 1 (sd 2, hereafter) contained not only $\mathrm{Chx} 10^{+}$and 

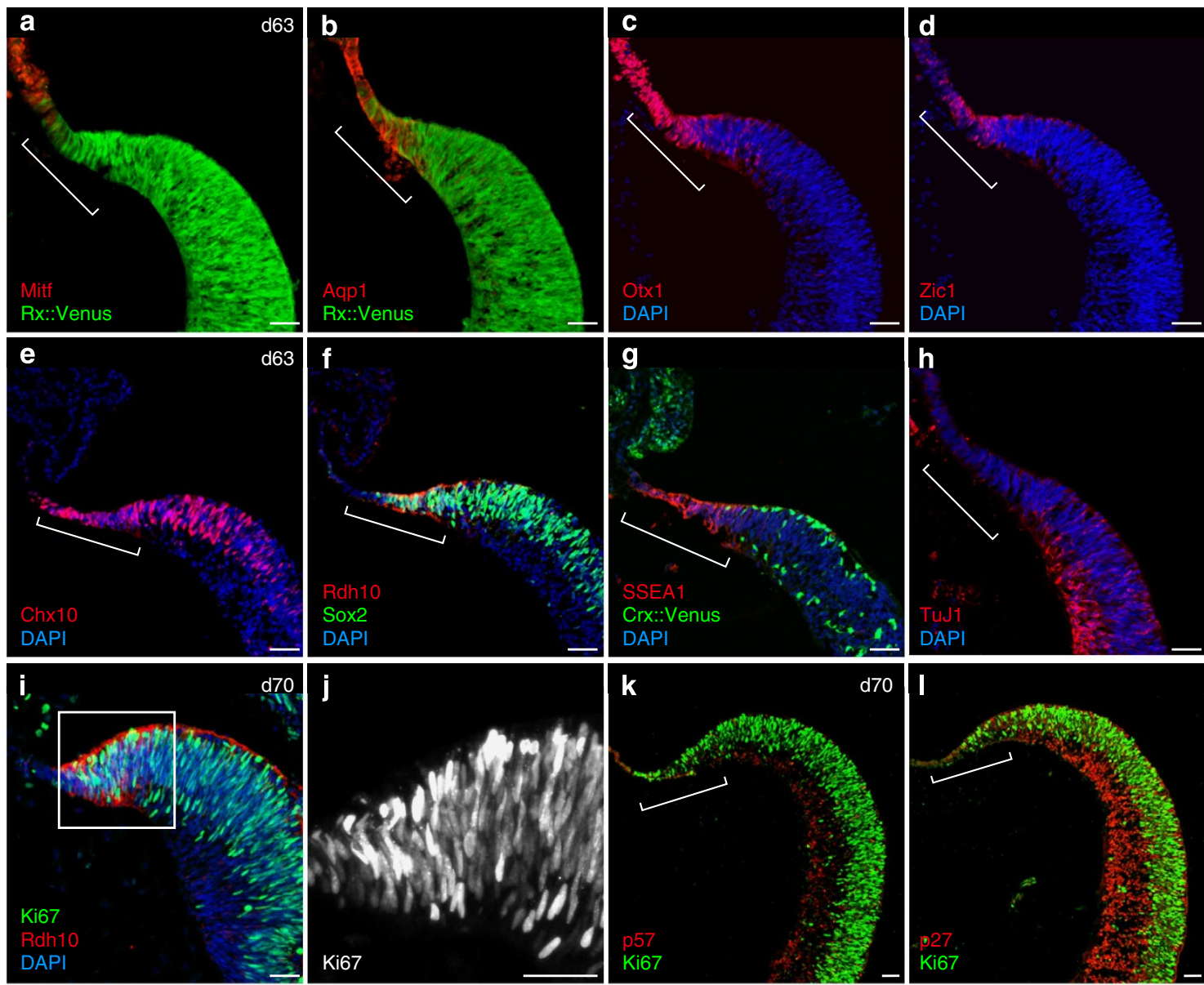

m

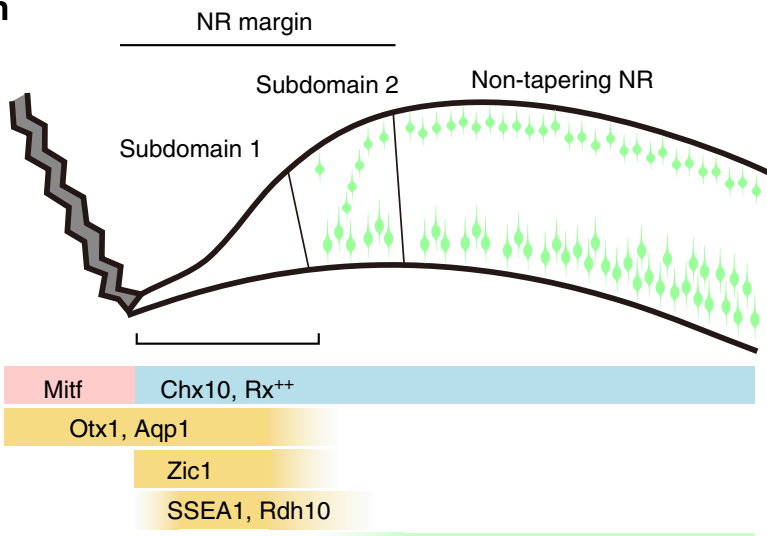

p27, p57

Crx, Brn3, Calretinin, TuJ1

Figure 4 | Subdomain-specific expression of CM markers in the NR margin. (a-I) Immunostaining analysis of CM-related markers in the NR margin of hESC-derived retina generated by the induction-reversal method: Mitf (a), Aquaporin1 (b), Otx1 (c), Zic1 (d), Rx::Venus (a,b), Chx10 (e), Sox2 (f), Rdh10 (f,i), SSEA1 (g), Crx::Venus (g; strong expression in photoreceptor precursors and week expression in early RPE), TuJ1 (h), Ki67 (i-I), p57 (k) and p27 (I). (a-h) Day 63. (i-I) day 70. Bracket represents CM-like zone. (j) High magnification of the boxed area in i. (m) Schematic of marker localization in the CM-like zone around the NR-RPE junction. Scale bars, $50 \mu \mathrm{m}$ (a-I). See also Supplementary Fig. 4.

Sox $2^{+}$progenitors (Fig. 4e,f), but also the fate-specific NR cell markers (Fig. 4g,h), particularly on its distal side. Conversely, no expression of Otx1, SSEA1, Zic1 and Rdh10 was observed in this region.

While both sd 1 and sd 2 in the tapering proximal NR contained $\mathrm{Ki}^{+} 7^{+}$mitotic cells (Fig. 4i,j), the two subdomains differed in the expression of CDK inhibitors (p21, p27 and p57), which positively control cell cycle exit ${ }^{50,51}$; sd 1 was mostly negative for p27 and p57, while sd 2 contained substantial numbers of cells positive for these CDK inhibitors (Fig. 4k,l). Expression of p21 was observed in neither subdomain (Supplementary Fig. 4e-g).

Together, these findings (summarized in Fig. $4 \mathrm{~m}$ ) indicate that the tapered epithelium portion adjacent to the NR-RPE junction, sd 1, has an expression profile resembling that of the in vivo $\mathrm{CM}$ zone. 
Sphere-forming progenitors from CM-like zones. We next examined whether the hESC-derived CM-like region, like the foetal CM, contains sphere-forming progenitors (Fig. 5a). We manually dissected nonpigmented $R x:$ :Venus-strong tissues in the tapered NR margin (CM-like tissues, hereafter) from hESCderived retina (day 60-85), dissociated them, and then subjected them to retinosphere culture $(1,000 \text { cells per well })^{17,52,53}$. For comparison, we dissected nonmarginal NR (central NR) tissues from the same aggregates.

While $R x:$ :Venus ${ }^{+}$primary retinospheres formed from both CM-like and central-NR groups (Fig. 5b,c; note that non-CMtype NR progenitors were also present in the central-NR tissues), the efficacy was higher with cells from CM-like tissues (Fig. 5d and Supplementary Fig. 5a). Secondary sphere formation $(1,000$ cells per well) also showed a clear difference in efficacy (Fig. 5e; central-NR group <CM-like group). Furthermore, the sizes of both primary and secondary spheres from CM-like tissue were significantly larger than those from central NR (Fig. 5b,f,g and Supplementary Fig. 5b). The majority of cells in the primary and secondary retinospheres from CM-like tissues were $\mathrm{Chx} 10^{+}$ progenitors (Fig. $5 \mathrm{c}$ ). These findings suggested that the CM-like tissue contains self-renewing progenitors with a robust proliferative capacity.

To confirm that sd 1 cells in the CM-like tissue have the sphereforming ability, we next examined the activity of the SSEA ${ }^{+}$cells purified from hESC-derived CM-like tissues (Fig. 5h-j and Supplementary Fig. 5c,d). SSEA ${ }^{+}$cells (purified by fluorescentactivated cell sorting (FACS) or magnetic-activated CS (MACS)) formed retinospheres (Fig. 5h) more frequently than did SSEA1 ${ }^{-}$ cells (Fig. 5i; MACS tended to have a better cell viability than FACS; Supplementary Fig. 5d). In addition, spheres from SSEA $1^{+}$ cells were substantially larger in size than those from SSEA1 ${ }^{-}$ cells (Fig. 5j). Although SSEA1 ${ }^{-}$cells may still have the potential to form retinospheres, these findings indicate that SSEA1 ${ }^{+}$cells play a major role in sphere formation from CM-like tissues.

When cultured under differentiation conditions (adhesion culture) for 2 weeks, retinospheres from CM-like tissues (both primary and secondary) produced cells expressing fate-specific retinal cell markers such as Crx/Blimp1 (photoreceptors), TuJ1/ Pax6 (ganglion cells) and Calretinin (ganglion cells and interneurons; Fig. 5k and Supplementary Fig. 5e). The generation of fate-specific retinal cells from spheres was also observed with SSEA1-sorted cells after 2-week culture (Supplementary Fig. 5f), while few differentiated cells were seen initially. Taken together, these observations indicate that the NR-RPE junction zone of hESC-derived retina not only has a characteristic CM expression profile, but also contains sphere-forming progenitors resembling CM stem cells.

De novo NR generation at hESC-derived CM-like zones. We next investigated the progenitor dynamics at the CM-like zone of hESC-derived retina, focusing on de novo generation of NR tissue at its margin. To this end, we performed pulse-labelling experiments using nucleotide analogues (BrdU and EdU; Fig. 6). One-day exposure of hESC-derived retina to BrdU heavily labelled most cells in sd 1 (Fig. 6a) and a majority of cells in sd 2. These cells expressed the mitotic marker Ki67 (Fig. 6a, top). When the pulse-labelled tissues were cultured without BrdU for an additional 6 days, the cells in sd 1 rarely retained BrdU labelling (Fig. 6b, sd 1), suggesting that sd 1 mainly contains actively proliferating progenitors in which BrdU was diluted by cell division (the loss of BrdU ${ }^{+}$cells was unlikely to be caused by selective cell death, since this subdomain showed no substantial accumulation of cleaved caspase 3, a marker for apoptosis; Supplementary Fig. 6a).
In contrast, BrdU-labelled Ki67-negative cells, representing newly born postmitotic cells, appeared in sd 2 (red cells in Fig. 6b, sd 2). Next, we cultured the tissue for 13 days after 1-day BrdU labelling and then treated with EdU for 1 day (Fig. 6c). In this case too, EdU uptake was seen in most $\mathrm{Ki}^{+} 7^{+}$cells in both sd 1 and sd 2. In contrast, BrdU label-retaining cells were found in the central side of sd 2, but rarely in sd 1 or the peripheral side of sd 2 (Fig. 6c).

After the tissue was further cultured for an addition 13 days, BrdU-retaining cells were found in the thick portion of the NR but rarely in sd 1 and sd 2, while EdU-retaining cells were seen also in sd 2 (Fig. 6d). These EdU-retaining cells expressed fate-specific markers such as Brn3, Crx and Calretinin (Supplementary Fig. 6b-d).

Collectively, these findings demonstrate dynamic generation and differentiation of progenitors in distinct subdomains around the CM-like zone of the hESC-derived retina (Fig. 6e). In sd 1, the epithelium consists mostly of actively proliferating $\mathrm{Ki}^{+} 7^{+}$cells and the nucleotide analogue labels in these cells are diluted fast by continuous cell division, suggesting that this is a place for the de novo production of progenitors from stem cells rather than a site for generation of postmitotic cells. In contrast, sd 2 have $\mathrm{Ki}^{+}{ }^{+}$ cells predominantly on the apical side and gradually accumulates Ki67 ${ }^{-}$postmitotic cells on the basal side as it goes farther from the NR-RPE junction, suggesting that postmitotic cells are generated in this subdomain, unlike sd 1. This idea is consistent with the observation that the CDK inhibitors p27 and p57 are expressed in sd 2 but rarely expressed in sd 1 (Fig. 4k,1 and Supplementary Fig. 4b-d). Within sd 2, label-retaining cells gradually disappear from the peripheral to central direction during a time course of 1-4 weeks, such that cells labelled earlier are found more distally from the NR-RPE junction. Taken together with the observation that sphere-forming SSEA1 ${ }^{+}$cells are densely present in sd 1 (Figs $4 \mathrm{~g}$ and $5 \mathrm{i}$ ), these findings indicate that the hESC-derived CM-like tissue constitutes a growth zone for de novo generation of retinal progenitors at the NR-RPE boundary in self-organizing retinal culture (Fig. 7).

\section{Discussion}

A long-standing question regarding the formation of the human eye is how new cells-both progenitors and postmitotic-are added to the rapidly expanding NR during foetal development. Previous studies in nonhuman model systems, have suggested that the CM of the eye can in certain situations function as a source of multipotent progenitor cells. Determining whether this is the case in human foetal eyes, however, has been difficult due to the limited number of experimental models amenable to investigating human NR growth, expansion and cell contribution.

In this study, to overcome this limitation, we have successfully applied the self-organization culture approach of human ESCs to recapitulate $\mathrm{CM}$ development in vitro. Using induction-reversal culture combined with BMP4-triggered highly selective retinal differentiation, we demonstrated two aspects of retinal selforganization in 3D hESC culture (Fig. 7): central-peripheral selfpatterning of NR and self-formation of CM-like growth zones. These two aspects may be closely related: the CM-like growth zone formation occurs in the tapered portion of the NR, which resembles many of the characteristics of the peripheral domain of the embryonic NR.

Thus, the presence of tissue interactions between the NR and RPE appear to foster the self-organization of stem cell nichecontaining CM-like growth zones. Taken together with our previous studies ${ }^{30,32}$, we now demonstrate that self-organizing retinal culture of hESCs is capable of not only generating highly ordered epithelial structures, but also orchestrating bona fide 
a

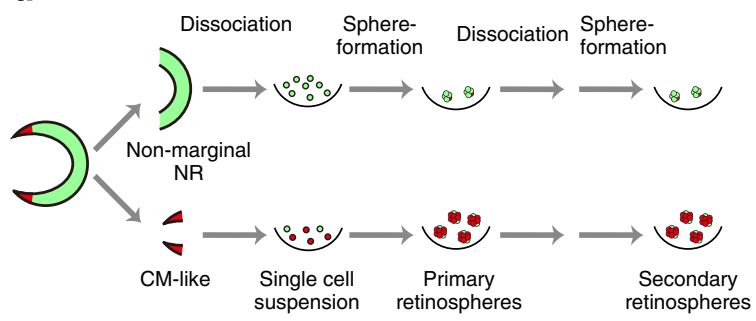

b

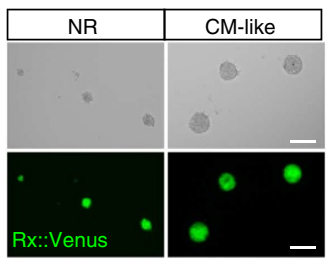

C

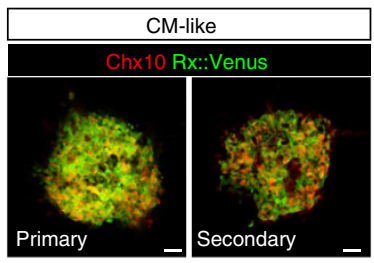

d

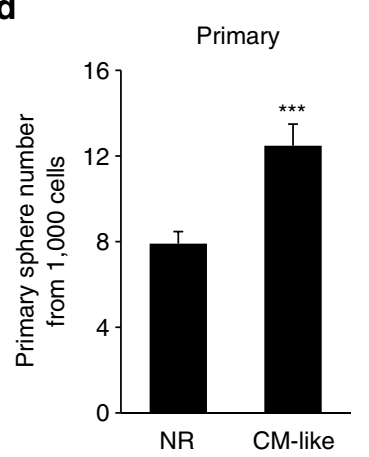

h

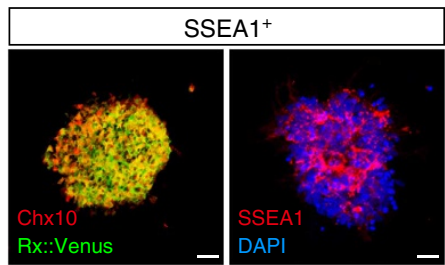

e

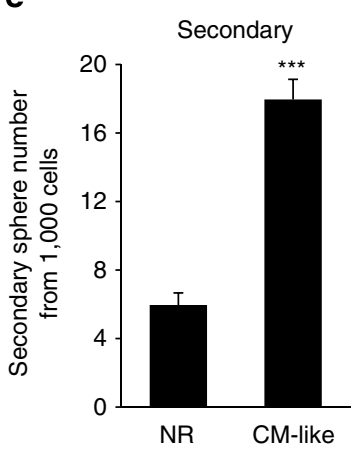

f

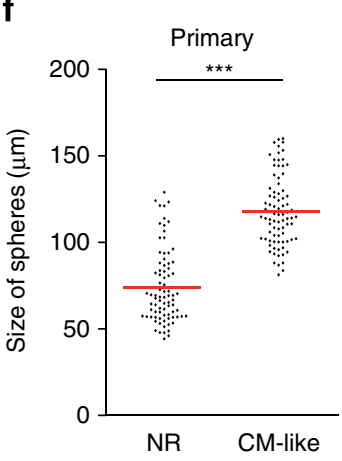

g

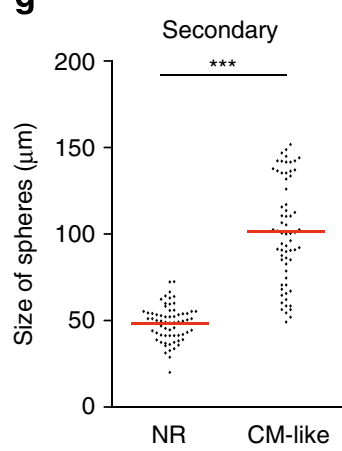

i

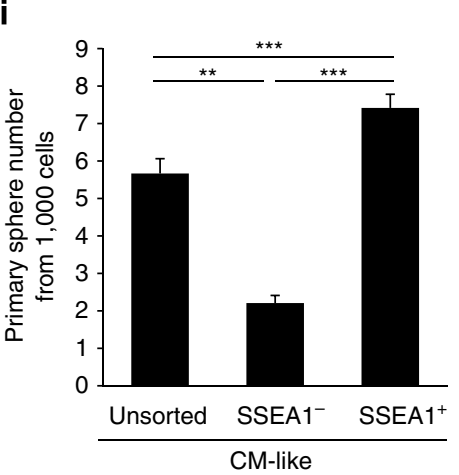

j

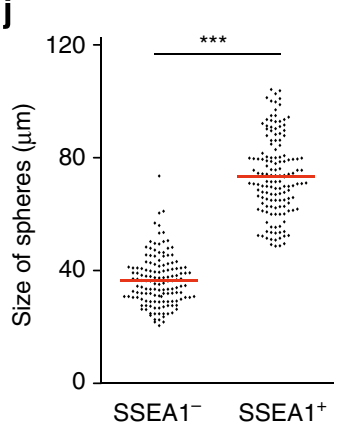

k

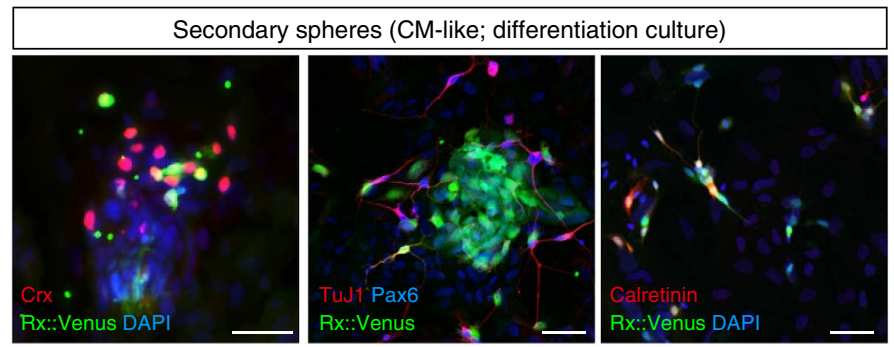

Figure 5 | Retinosphere formation from cells of CM-like tissues. (a) Schematic of retinosphere formation assays of cells from hESC-derived NR.

(b) External appearance of primary spheres formed from dissociated cells of CM-like (right) and nonmarginal NR (left) tissues of hESC-derived NR (day 85). (c) Expression of Rx::Venus and Chx10 in primary and secondary spheres forming from dissociated cells of CM-like tissues of hESC-derived NR (day 70). (d) Number of primary spheres forming from 1,000 dissociated cells of these tissues (day 70). Data represent mean \pm s.e.m. of 12 experiments (each experiment with 8 samples; counted spheres, NR =759, CM-like =1,198). (e) Number of secondary spheres forming from 1,000 dissociated cells of corresponding primary spheres (formed from tissues on day 70 and cultured under sphere-forming conditions for 14 days). Data represent mean \pm s.e.m. of 6 experiments (each experiment with 8 samples; counted spheres, NR $=286, C M$-like $=862$ ). $(\mathbf{f}, \mathbf{g}$ ) Sizes (maximum diameters) of forming primary (f) and secondary (g) spheres. Red lines represent the mean sizes. (h) Immunostaining of retinospheres forming in dissociation suspension culture of FACS-sorted Rx::Venus ${ }^{+}$SSEA $1+$ cells from hESC-derived CM-like tissues. (i) Number of spheres forming from CM-like tissues (unsorted), MACS-purified SSEA $1^{+}$cells $\left(S S E A 1^{+}\right)$and SSEA $1^{-}$cells $\left(S S E A 1^{-}\right)$, which were sorted from day 79 and plated at the density of 1,000 cells per well. Data represent mean \pm s.e.m. of 6 experiments (each experiment with 8 samples; counted spheres, unsorted $=272$, SSEA $^{-}=106$, SSEA $^{+}=356$ ). (j) Size of spheres from FACS-purified $R x:: V_{\text {enus }}{ }^{+}$SSEA ${ }^{+}$cells and $R x:: V e n u s{ }^{+}$SSEA $1^{-}$cells. Red line represents the mean size. (k) Expression of fate-specific cell markers in differentiation culture of CM-like-tissue-derived secondary spheres. (d,e) ${ }^{\star \star \star} P<0.001$. Student's $t$-test. (i) ${ }^{\star \star} P<0.01$. ${ }^{\star \star \star} P<0.001$. Analysis of variance followed by post-hoc Tukey's test. (f,g,j) ${ }^{\star \star \star} P<0.001$. Mann-Whitney's test. Scale bars, $100 \mu \mathrm{m}(\mathbf{b}), 50 \mu \mathrm{m}(\mathbf{k})$ and $25 \mu \mathrm{m}(\mathbf{c}, \mathbf{h})$. See also Supplementary Fig. 5. 
a
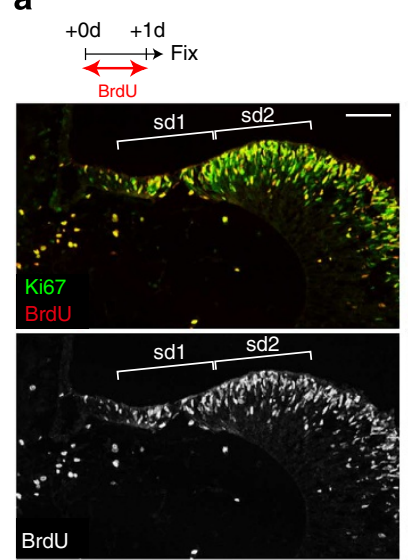

C
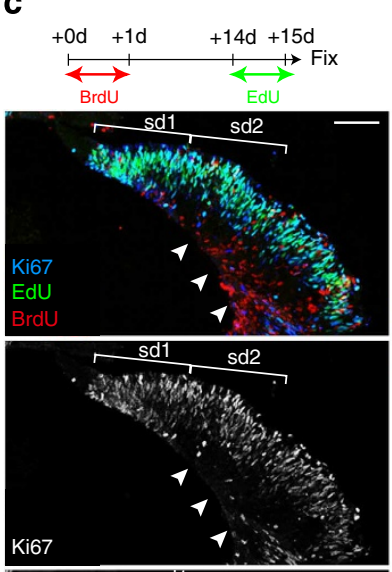

Ki67
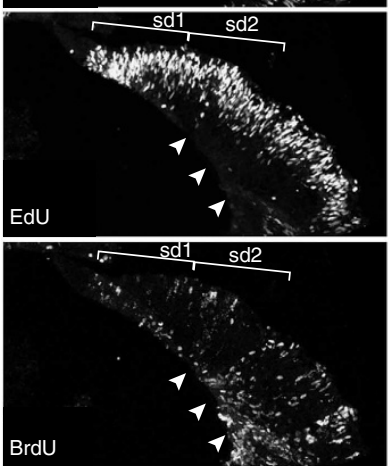

b

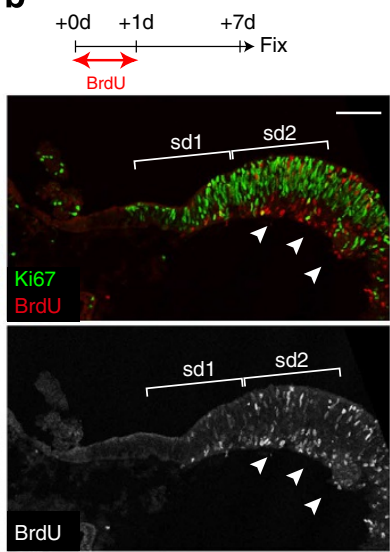

d
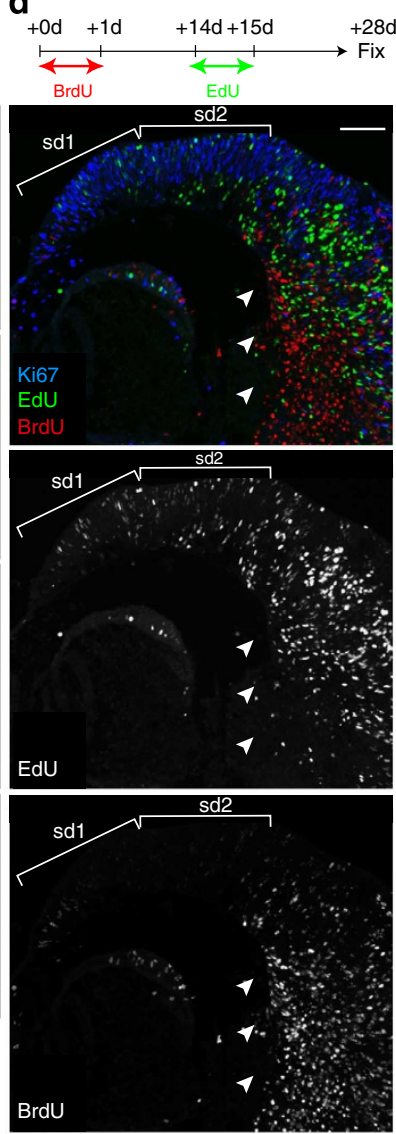
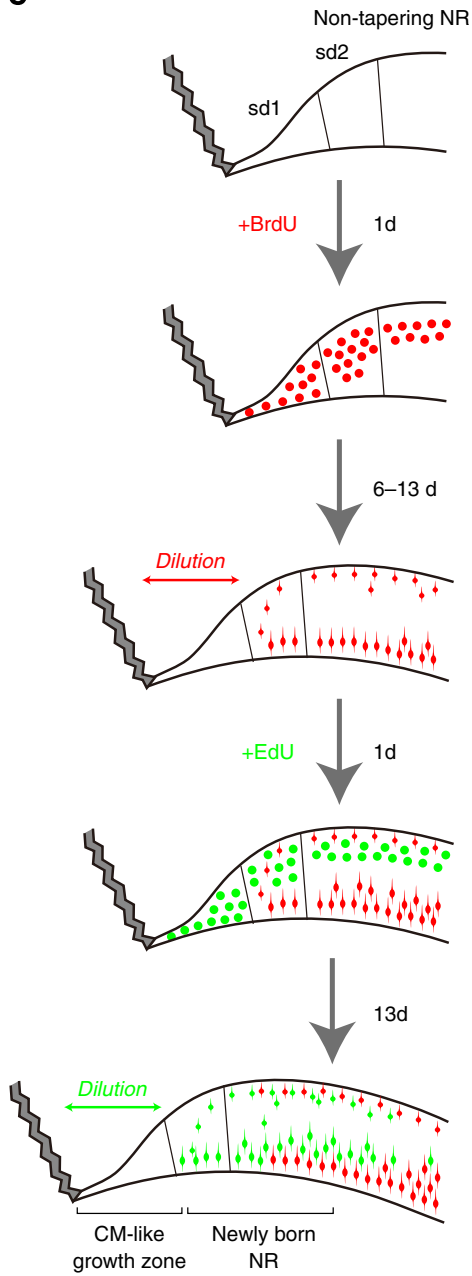

Figure 6 | CM-like subdomain in hESC-derived NR functioning as a growth zone. (a) Distribution of BrdU incorporation (red) after a 1-day BrdU exposure (starting at day 60). Immunostaining of Ki67, green. Note that most of the Ki67 ${ }^{+}$cells in subdomain 1 (sd 1), subdomain 2 (sd 2) and central NR are positive for BrdU. (b) Distribution of BrdU-retaining cells after an additional 6-day culture without BrdU. Note that most of Ki67+ cells in sd 1 and sd 2 are negative for BrdU (brackets) and most of BrdU ${ }^{+}$cells in basal side of sd 2 and central NR are negative for Ki67 (arrowheads). (c) Distribution of BrdUretaining cells (red) pulse labelled 2 weeks before (starting at day 60) and of EdU-labelled cells (green) by exposure over the last $24 \mathrm{~h}$. Note that most of the $\mathrm{Ki}^{+} 7^{+}$cells in sd 1 and sd 2 are $\mathrm{EdU}^{+} \mathrm{BrdU}^{-}$(brackets) and most of BrdU ${ }^{+}$cells in basal side of sd 2 and central NR are negative for Ki67 (arrowheads). (d) Distribution of BrdU-retaining cells (red) pulse labelled 4 weeks ago (starting at day 60) and of EdU-labelled cells (green) pulse labelled 2 weeks ago. Note that most of $\mathrm{Ki} 7^{+}$cells in sd 1 and sd 2 are negative for EdU nor BrdU (brackets) and most of BrdU ${ }^{+}$cells or EdU ${ }^{+}$cells in basal side of sd 2 and central NR are negative for Ki67 (arrowheads). (a-d) Brackets, sd 1 and sd 2. Scale bars, $100 \mu m$ (a-d). (e) Summary of results of BrdU/EdUlabelling analyses. See also Supplementary Fig. 6.

organogenesis allowing a stem cell system to maintain and expand the developing organ in vitro.

In induction-reversal culture, GSK3 and FGFR inhibitorinduced thin RPE tissue ( $R x::$ Venus-dim) exhibited a transition to $R x:$ :Venus-strong thick NR epithelium within a few days after exposing RPE tissue to NR-differentiation medium (Supplementary Movies 1 and 2). A similar induction-reversal phenomenon is also seen when only the GSK3 inhibitor was applied. However, the further addition of the FGFR inhibitor (which by itself had only weak effects if any) made RPE 

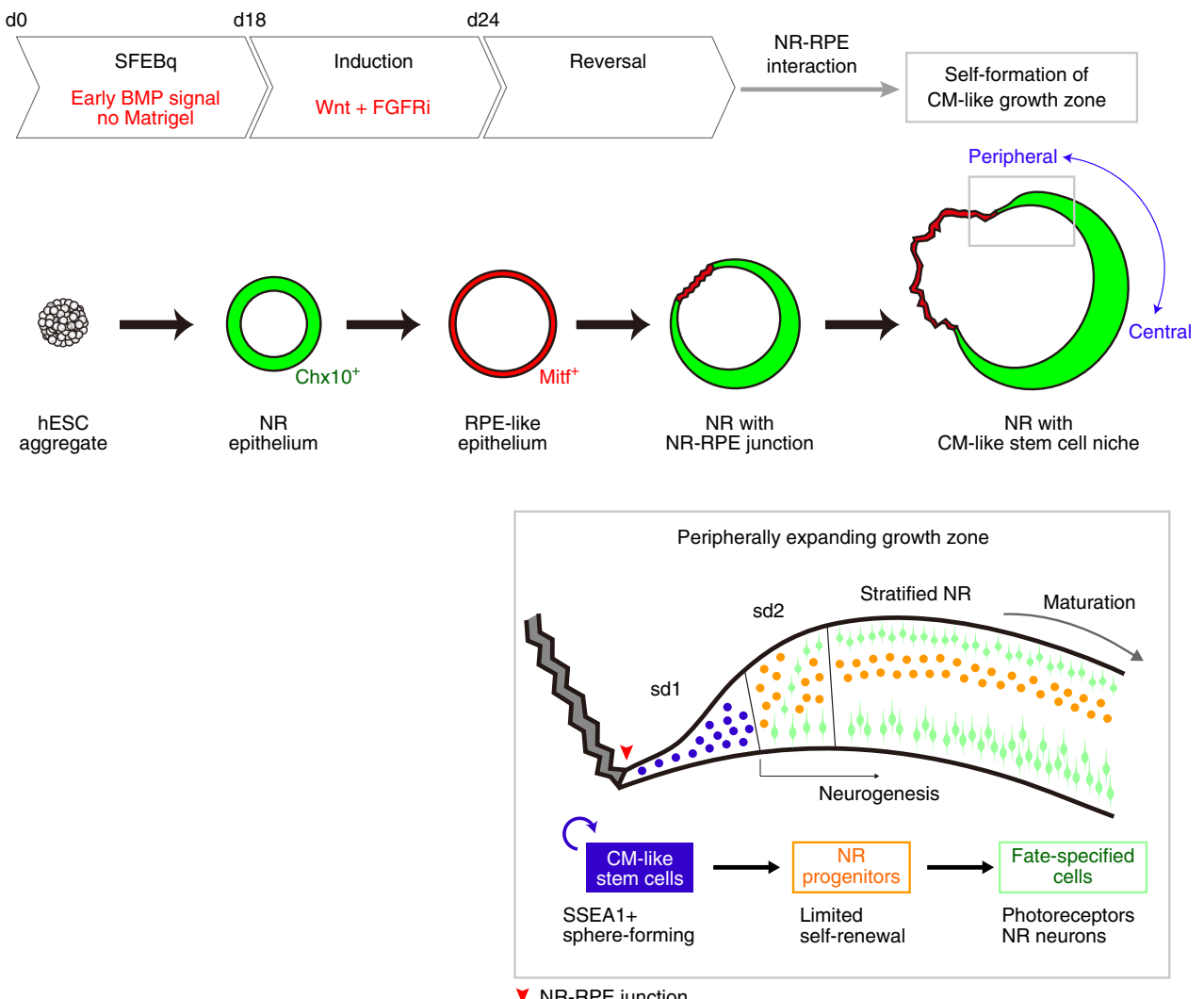

Figure 7 | Self-organization of growth zone with stem cell niche from hESCs. Schematic of self-organization culture of hESCs involving selective NR differentiation with transient BMP4 treatment, induction-reversal culture and self-formation of CM-like tissue at the NR-RPE boundary. Inset, schematic of a self-forming CM-like zone at the margin of hESC-derived NR with SSEA $1^{+}$sphere-forming stem cells (blue), which produces NR progenitors. The CM-like growth zone functions in a similar manner with the progress zone in the developing limb, with regard to self-renewal and tissue extension.

differentiation more complete in a relatively short period (see Methods for details). The combination of both inhibitors induces 'all-RPE' aggregates (with little NR-type epithelium) in $>90 \%$ of aggregates of 'induction' culture. After removal of the inhibitors in 'reversal' culture, approximately three quarters of aggregates develop a NR-dominant epithelia (Fig. 3e; NR only + two domain).

Live imaging indicated that the reappearance of NR-type epithelium in reversal culture is caused by conversion of RPE-type epithelium rather than by rapid expansion of residual NR-type epithelium in the aggregate. In addition, this 'RPE-to-NR' conversion, triggered only by a change in extracellular signals, can occur even after the onset of Mitf expression (bona fide RPE marker) and the repression of Chx10 expression (bona fide NR marker). These observations are consistent with previous studies in vivo showed a profound plasticity between the $\mathrm{NR}$ and RPE fates during development ${ }^{41,42,54}$. Interestingly, the temporal window of competence for RPE-NR reversal may be limited in our hESC culture; preliminary experiments have indicated that a longer treatment of GSK3 and FGFR inhibitors (for example, days 18-27 or 18-30, instead of days 18-24) makes RPE less responsive to reversal signals in NR-differentiation medium. This suggests that the length of RPE-specifying culture (which may deepen RPE commitment) affects the reversion efficacy. In addition, an intriguing report has described that a subpopulation of adult human RPE cells, on activation, gain competence to become multipotent stem cells ${ }^{55}$, suggesting that RPE cells, even in adult humans, maintain special state of epigenetic control. In future investigations, our culture method may be useful in elucidating molecular mechanisms for the stage- and species-specific control of retina transdifferentiation competence.

In our hESC culture, some variation was observed in the response to the reversal trigger at the tissue level. In $\sim 40 \%$ of aggregates, RPE and NR were co-generated (two-domain aggregates) and CM-like zones self-formed between them. In $\sim 30 \%$ of aggregates, most of epithelia became entirely NR after reversal, whereas $25-30 \%$ of aggregates were resistant to the reversal trigger and remained RPE dominant.

The cause for this heterogeneity in responsiveness is an intriguing question. We infer that it may be relevant to a 'bistability' control, which tends to give a 'black-or-white' (not grey) outcome (reviewed in ref. 56). In the signalling network for retinal specification (Supplementary Fig. 2), RPE differentiation is promoted by Wnt signals, and, once the retinal tissue becomes RPE, it enhances local Wnt signals by inducing endogenous expression of Wnts and Mitf $\mathrm{f}^{5,58}$. Conversely, NR differentiation is suppressed by Wnt signals ${ }^{37-40}$. Moreover, differentiating NR shuts off local Wnt signals by attenuating Wnt ligand production by the transcription factor Six3 (ref. 40) and secreting Wnt antagonists such as Dkk1 (refs 30,59). Furthermore, specification into NR and RPE is promoted and suppressed by FGF signals, respectively, which are produced by $\mathrm{NR}^{41}$. These multiplex regulations of mutual inhibition and autoactivation lead to a 'bistable' system, in which a small difference in endogenous signals could make a pivotal specification of retinal epithelium (or its local domains) into either NR or RPE. In the two-domain aggregate containing both NR and RPE, only a small portion of RPE is resistant to reversal signals promoting NR differentiation. We infer that a bias for the location of the RPE-prone domain 
may be related to the co-presence of nonretinal neural tissues (representing $\sim 10 \%$ of cells; Fig. 1n,o), which was previously shown to promote RPE differentiation within the ESC-derived optic cup ${ }^{30}$. Consistent with this idea, in close visual examinations of two-domain hESC aggregates after inductionreversal culture (Fig. 3c,d), nonpigmented $R x::$ Venus ${ }^{-}$tissues (that is, nonretinal neural tissues) were not found within the continuous NR but preferentially located together with pigmented RPE.

Another remaining question concerns how the boundary between RPE and NR is determined. In 'reversal' culture method, our preliminary observations suggest that thin RPE tissue (sometimes adjacent to nonretinal tissue) tends to resist the reversal stimuli, whereas the retinal epithelium distally (centrally) located from thin RPE tissue tends to exhibit a more rapid transition to strong $R x:$ :Venus-expressing thick NR epithelium. This raises a possibility that some bias in the differentiation state has already existed in RPE-like tissue before starting the reversal culture method. This hypothesis will be more rigorously tested in future studies using RPE/NR double-labelled cell lines, which might allow a more detailed observation of the dynamics of NR-RPE formation.

The 'induction-reversal culture' method enables the cogeneration of NR and RPE in hESC culture. In this continuous structure, the NR exhibits a central-peripheral polarity. With regard to morphology, a graded change in the epithelial thickness is seen along the central-peripheral (thick-to-thin) axis of hESCderived NR. In the central portion, NR differentiation into fatespecific cells tends to be more advanced than in the peripheral portion, while immature progenitors are more prominent in the NR periphery, particularly in the tapering domain where de novo NR generation occurs.

This self-formation of the central-peripheral polarity appears to correlate with the presence of RPE adjacent to the NR, since such polarity is not obvious within NR epithelia in hESC aggregates without RPE (in the latter case, the entire NR tends towards the central-NR type with regard to differentiation and thickness; Fig. 1q). Moreover, when NR epithelium, by chance, is flanked by more than one RPE domain, a CM-like zone emerges in the NR domain next to each NR-RPE junction. It remains to be clarified whether the effect of RPE is mediated by soluble signals or spreads via planar induction. Another related question for future study is whether the effect of RPE on the centralperipheral polarity in NR is direct on the NR or secondary through the CM-like zone formation between them.

In the human foetal eye, the regional difference along the central-peripheral axis is quite remarkable. For instance, the generation of morphologically recognizable photoreceptors is observed around foetal week 10 at the central-most NR region, while photoreceptor differentiation in the noncentral region occurs very gradually over the following 15 weeks and slowly proceeds in a central-peripheral pattern ${ }^{44,60}$. The central-most NR domain eventually gives rise to the fovea, in which photoreceptors are mostly cones. The generation of the fovea-like structure (a pit structure selectively expressing colour opsins and not Rhodopsin) has not been observed thus far in our culture yet, and is an intriguing and challenging topic for future study. As described in our previous report ${ }^{32}$, both RXR-gamma ${ }^{+}$cone precursors and $\mathrm{Nrl}^{+}$rod precursors are generated in wide domains of hESC-derived NR. Therefore, a first step towards this challenging goal may be region-selective control of cone and rod generation along the central-peripheral axis of the self-organized NR.

In induction-reversal culture, the tapered peripheral end of the hESC-derived NR (sd 1), adjacent to the $\mathrm{Mitf}^{+}$domain, formed a niche for $\mathrm{SSEA}^{+}$stem-like cells that continuously produce progenitors. Cells in sd 1 were $\mathrm{Ki}^{+}{ }^{+}$and were efficiently labelled with nucleotide analogues after 1-day of exposure, while these labels in sd 1 (showing no substantial level of apoptosis) became undetectable within 6 days, indicating that these cells are under active proliferation. sd 1 , unlike sd 2, contained few fatecommitted postmitotic cells and did not express CDK inhibitors (promoting cell cycle exit), suggesting that these actively proliferating cells in sd 1 were not directed to produce terminally differentiated postmitotic cells by cell cycle exit.

SSEA $1^{+}$cells are also present in the CM-like zone of the mouse foetal retina. However, the strict identity of SSEA $1^{+}$cells across species could be a matter of argument. For instance, during early mouse retinal development, SSEA1 is widely expressed throughout the optic vesicle. From E13.5 onwards, SSEA1 expression is mainly seen in the peripheral NR and pigmented outer layer of the ciliary epithelium ${ }^{48}$. However, within the mouse peripheral NR, the nonpigmented epithelium at the peripheral end (corresponding to sd 1) is devoid of SSEA1 expression, in contrast to our finding with its expression in the CM-like tissue generated from hESCs. Since early SSEA1 expression in the mouse retina dynamically changes in a stage-specific manner ${ }^{48}$, a careful stage-by-stage investigation would be necessary to examine whether SSEA1 ${ }^{+}$cells in the mouse and human foetal retinae represent distinct (albeit related) subpopulations in the growing end of the tissue.

Clarke et al. recently reported the generation of 'retinal stemlike cells' from hESCs, but these stem-like cells may have critical differences from those generated in our study. Namely, the stemlike cells reported by Clarke et al. ${ }^{53}$ were derived from a rare population within hESC-derived pigmented cells (stem cells from pigmented ciliary epithelial cells are also described in ref. 61). In contrast, the retinospheres in the present study are derived from retinal tissues from which pigmented tissues were removed. Moreover, the SSEA1 ${ }^{+}$cell population did not show obvious pigmentation in the tissue section nor by visual inspection of dissociated immunostained cells (Supplementary Fig. 5g; they did not show strong side scattering in FACS analysis either; not shown). Thus, the stem cell population in the CM-like zone of our hESC culture (within the Chx10 ${ }^{+}$peripheral NR) appears to be distinct from the one reported previously, which seems to be more closely related to RPE or pigmented ciliary epithelium. We infer that the 3D formation of retinal tissue with juxtaposed NR and RPE is vital for the generation of CM-type stem cells, which contribute to de novo NR extension in the peripheral direction.

Regarding human retinal stem cells, another set of previous studies have suggested the presence of Müller glia-type stem cells in the marginal zone of human adult retina ${ }^{19,62}$. The similarity and difference between these adult-type cells and CM-type stem cells in our hESC culture (presumably foetal type) is another intriguing topic for future investigation. In the human foetus, the $\mathrm{CM}$ appears by foetal week 10 and the ciliary body formation begins at the end of the 3 rd foetal month ${ }^{45}$. We have so far failed to observe the morphological appearance of ciliary body-like structures in our hESC culture, whereas $\mathrm{Mitf}^{+} / \mathrm{Aqp} 1^{+}$epithelia (Fig. 4), reminiscent of ciliary epithelium, was readily generated.

While the present study focused on new aspects of selforganization of hESC-derived retina, the improved culture that is introduced here also serves as an advanced protocol towards future applications of human pluripotent stem cells. First, the improved culture has enabled a more selective retinal differentiation of hESCs $(>80 \%)$ by the use of timed BMP4 treatment. In our preliminary experiments, other BMPs such as BMP2, BMP7 and Gdf7 had similar inductive abilities. Moreover, unlike our and others' previous methods, this BMP-based hESC culture does not require the xenogenic matrix protein mixture Matrigel for the $3 \mathrm{D}$ formation of NR. The precise mode of BMP4 action in retinal 
specification remains an intriguing unanswered question. Previous studies have shown that BMP signals directly suppress the expression of Foxg1, which is essential for cortical development ${ }^{63,64}$. Therefore, one interpretation may be that BMP4 treatment during the early differentiation stage in our culture prevents hESC-derived forebrain neuroepithelium from adopting the telencephalic fate and redirects it into retinal progenitors. In the early embryo, BMP signals are expressed near the anterior neural ridge and in the prechordal mesoderm, and are implicated in the patterning of rostral diencephalon of the early embryo ${ }^{65}$, including hypothalamic (diencephalic) induction 66,67

In condition 1 of our new culture method, the continuous NR forms vesicles in apically convex manner, which resemble retinal tissues shown by Meyer et al, 2011 (ref. 27). The vesicles formed in our culture, however, do not undergo further morphogenesis (invagination) to form optic cup structures, since they lack RPE as the outer wall. Regarding the long-term culture, after 60 days, the NR in vesicles gradually looses its integrity and tends to accumulate neural rosettes (differentiation itself proceeds).

In the induction-reversal culture combined with BMP4induced retinal differentiation (condition 3), a portion of RPE tissue forms adjacent to NR (Fig. 3c,d). In this two-domain aggregate, NR is generated as a large continuous epithelium. NR can be also obtained from optic cups cultured in hESC aggregates under our previous conditions ${ }^{32}$. However, our new culture method has clear advantages in the reproducible generation of larger NR epithelia. First, the formation of optic cups in our previous culture was not very frequent $(\sim 10 \%)$. Second, NR epithelia in these optic cups were typically $500 \mu \mathrm{m}$ in diameter around day 35 . In contrast, NR forms as a fairly large domain (typically $1.3-1.5 \mathrm{~mm}$ in diameter at day 35; Fig. 3c) with induction-reversal culture, spanning a majority of the aggregate surface, and continuous NR epithelium grows robustly (for example, typically $2 \mathrm{~mm}$ at day 60; Fig. $3 \mathrm{~d}$ ).

Finally, the two-domain structure of NR epithelium has a characteristic thick and transparent appearance, and its boundary to RPE can be easily recognized because of the presence of pigmented tissue adjacent to it. This simple structure may facilitate the preparation of high-purity NR tissues in large quantities (much like removing the stem ends from radishes) and may prove useful in future studies examining optic tissue transplantation, pathogenesis and drug discovery.

\footnotetext{
Methods

Maintenance of hESCs. hESCs (KhES-1) were used according to the hESC research guidelines of the Japanese government. hESC reporter lines ( $R x::$ Venus, $R x::$ AcGFP and $C r x::$ Venus) were generated by using Neon Transfection System (Life Technologies) $^{32}$. hESCs were maintained on a feeder layer of MEFs inactivated by mitomycin C treatment in DMEM/F12 (Sigma; cat \# D6421; suitable for $2 \% \mathrm{CO}_{2}$ culture) supplemented with $20 \%$ (vol/vol) KSR (Gibco), $2 \mathrm{mM}$ glutamine, $0.1 \mathrm{mM}$ nonessential amino acids (Gibco), $8 \mathrm{ng} \mathrm{ml}^{-1}$ recombinant human bFGF (Wako), $0.1 \mathrm{mM} 2$-mercaptoethanol, $50 \mathrm{U} \mathrm{ml}^{-1}$ penicillin and $50 \mu \mathrm{g} \mathrm{ml}^{-1}$ streptomycin under $2 \% \mathrm{CO}_{2}$ conditions. For passaging, hESC colonies were detached and recovered en bloc from the feeder layer by treating them with $0.25 \%$ trypsin and $1.0 \mathrm{mg} \mathrm{ml}^{-1}$ collagenase IV in PBS containing $20 \% \mathrm{KSR}$ and $1 \mathrm{mM} \mathrm{CaCl}{ }_{2}$ at $37^{\circ} \mathrm{C}$ for $7 \mathrm{~min}$. The detached hESC clumps were broken into smaller pieces (several dozens of cells) by gentle pipetting. The passages were performed at a 1:3 to $1: 5$ split ratio every 3 rd or 4 th day.
}

Retinal differentiation from hESCs. For serum-free floating culture of embryoid body-like aggregates with quick reaggregation (SFEBq) culture, hESCs were dissociated into single cells in TrypLE Express (Gibco) containing $0.05 \mathrm{mg} \mathrm{ml}^{-1}$ DNase I (Roche) and $20 \mu \mathrm{M}$ Y-27632, and quickly re-aggregated using low-celladhesion 96-well plates with V-bottomed conical wells (Sumilon PrimeSurface plate; Sumitomo Bakelite) in 'differentiation medium' (12,000 cells per well, $100 \mu \mathrm{l}$ ) supplemented with $20 \mu \mathrm{M}$ Y-27632 (ref. 68) under $5 \% \mathrm{CO}_{2}$ at $37^{\circ} \mathrm{C}$. The differentiation medium is gfCDM ${ }^{69}$ supplemented with $10 \% \mathrm{KSR}$, while gfCDM contains 45\% Iscove's modified Dulbecco's medium (IMDM, Gibco), 45\% Hams F12 (F12, Gibco), Glutamax, 1\% chemically defined lipid concentrate (Gibco), monothioglycerol $\left(450 \mu \mathrm{M}\right.$, Sigma), $100 \mathrm{U} \mathrm{ml}^{-1}$ penicillin and $100 \mu \mathrm{g} \mathrm{ml}^{-1}$ streptomycin (Gibco). Defining the day on which the SFEBq culture was started as day 0 , recombinant human BMP4 (R\&D) was added to the culture to the final $1.5 \mathrm{nM}(55 \mathrm{ng} / \mathrm{ml})$ on day 6 , and its concentration was diluted into half by half medium change every 3 rd day. The addition of BMP4, started on day 3 and day 6 , induced $\mathrm{Rx}^{+} \mathrm{NR}$ epithelium at day 18 with similar efficiency. The number of initial plating of cells (12,000 cells per well) was optimized (in both efficiency and size), but $\mathrm{Rx}^{+} \mathrm{NR}$ epithelium was also achieved using 9,000 cells per well.

To establish NR-differentiation culture from hESCs without the use of Matrigel, we examined the effects of the following signal modulators on the induction of $R x:: V_{\text {Venus }}{ }^{+}$cells at various doses and time points: BMP agonists/antagonists (BMP4, dorsomorphin), Wnt agonists/antagonists (Wnt3a, IWR1e), Shh agonists/ antagonists (SAG, cyclopamine) and FGF agonists/antagonists (FGF2, SU5402). Among them, the addition of BMP4 at low concentrations during early phases of SFEBq culture (day 3 or day 6 ) proved to most effectively induce $R x:$ :Venus ${ }^{+}$ retinal epithelium on day 24 .

Long-term NR culture. NR epithelium was subjected to long-term culture under the following conditions: on day 18, NR-containing aggregates were transferred from a 96-well plate to a 9-cm Petri dish (noncell adhesive; Sumitomo Bakelite) and further cultured in suspension (60 aggregates/9-cm dish for days 18-50; 30 aggregates after day 50) under $40 \% \mathrm{O}_{2} / 5 \% \mathrm{CO}_{2}$ conditions in 'NR-differentiation medium' containing DMEM/F12-Glutamax medium (Gibco), 1\% N2 supplement (Gibco), 10\% foetal bovine serum (FBS), $0.5 \mu \mathrm{M}$ retinoic acid (Sigma), $0.1 \mathrm{mM}$ taurine (Sigma), $0.25 \mu \mathrm{g} \mathrm{ml}^{-1}$ Fungizone (Gibco), $100 \mathrm{U} \mathrm{ml}^{-1}$ penicillin and $100 \mu \mathrm{g} \mathrm{ml}^{-1}$ streptomycin. NR tissues continuously grew under these conditions for the next several weeks, at least. RPE tissues grew slowly compared with NR tissues.

Induction-reversal culture. To induce CM-like tissues within NR, NR epithelium generated from hESCs was subjected to two-step 'induction-reversal' culture under the following conditions. For the 'induction' culture, NR-containing aggregates generated from hESCs on day 18 were transferred from a 96 -well plate to a $9-\mathrm{cm}$ Petri dish (noncell adhesive), and further cultured in suspension for 4-6 days under $5 \% \mathrm{CO}_{2}$ conditions in DMEM/F12-Glutamax medium containing $1 \% \mathrm{~N} 2$ supplement, $3 \mu \mathrm{M}$ CHIR99021 (GSK3 inhibitor; Stemgent), $100 \mathrm{U} \mathrm{ml}^{-1}$ penicillin and $100 \mu \mathrm{g} \mathrm{ml}^{-1}$ streptomycin. The addition of $5 \mu \mathrm{M}$ SU5402 (FGFR inhibitor; Sigma) treatment to this induction medium increased efficiency and reproducibility of RPE induction and formation of CM-like tissues after the reversal culture, while CHIR99021 treatment without SU5402 tended to give higher variations in the level of RPE induction.

For the 'reversal' culture, the floating aggregates with RPE-like thin epithelium (Fig. 2b,c) were cultured in suspension under $40 \% \mathrm{O}_{2} / 5 \% \mathrm{CO}_{2}$ conditions in NRdifferentiation medium (described above). On day 50, two-domain aggregates with CM-like tissues formed and they were further cultured in NR-differentiation medium. Two-domain aggregates with CM-like tissues stably grew in long-term culture. For the reversal culture, the addition of FBS is essential and its depletion from the reversal culture medium substantially decreased NR differentiation.

CM-like tissues could be also generated by the induction-reversal culture using NR epithelia obtained by our previous method using Matrigel ${ }^{32}$. CM-like tissues generated by using Matrigel and induction-reversal culture were similar to those generated by using induction-reversal culture with BMP4 treatment, as judged by CM marker expression and morphology (data not shown). NR epithelia generated with Matrigel tended to be more sensitive to RPE-induction medium than BMP4induced NR and more reproducibly underwent RPE differentiation in medium containing CHIR99021.

Both our previous method ${ }^{32}$ (Matrigel based) and new method (BMP treatment) showed a similar time course of differentiation: Brn3b ${ }^{+}$cells $(\sim \mathrm{d} 28)$, $\mathrm{Crx}^{+}$cells $(\sim \mathrm{d} 35)$, Recoverin ${ }^{+}(\sim \mathrm{d} 45), \mathrm{RXRG}^{+}$cells $(\sim \mathrm{d} 60), \mathrm{NRL}^{+}$cells $(\sim \mathrm{d} 100), \mathrm{S}_{-}$opsin ${ }^{+}$cells $(\sim \mathrm{d} 130)$ and Rhodopsin ${ }^{+}$cells (weak expression, $\sim$ d130). $\mathrm{CRALBP}^{+}$or GFAP ${ }^{+}$cells (Muller glia) and PKC-alpha ${ }^{+}$cells (bipolar cells) were not yet detected $(\sim \mathrm{d} 140)$. When we applied the 'induction-reversal' methods, differentiation of these markers tended to be delayed by several days (corresponding to the time for RPE-induction phase). NR progenitors, generated by BMP treatment with 'induction-reversal' method, differentiated into Crx ${ }^{+}$ photoreceptors by DAPT (Sigma) treatment for 7 days (Supplementary Fig. 7) as described previously ${ }^{32}$. Floating aggregates and retinospheres were analyzed with a fluorescence microscope (KEYENCE, BIOREVO).

Retinosphere formation and differentiation. For sphere formation assays, aggregates with CM-like tissues (day 60-90) were manually dissected into CM-like tissues (tapering NR without pigmented RPE tissue) and nonmarginal NR tissues (nontapering central NR) using fine forceps under stereo microscope (Olympus, SZX16). After digestion in papain (Sumitomo Bakelite) at $37^{\circ} \mathrm{C}$ for $30 \mathrm{~min}$, both tissues were gently dissociated into single cells by pipetting. Dissociated single cells were plated on 96-well flat bottom plates (MPC coated; Nunc) at a density of $1,000-3,000$ cells per well $\left(0.5-1.5 \times 10^{4}\right.$ cells per $\left.\mathrm{ml}\right)$ and cultured for 7 to 14 days 
in 'retinosphere medium' containing DMEM/F12-Glutamax medium, 2\% B27 supplement (without vitamin A; Gibco), $20 \mathrm{ng} \mathrm{ml}^{-1}$ human bFGF, $20 \mathrm{ng} \mathrm{ml}^{-1}$ human EGF (R\&D), $5 \mu \mathrm{g} \mathrm{ml}^{-1}$ heparin (Sigma), $0.25 \mu \mathrm{g} \mathrm{ml}^{-1}$ Fungizone, $100 \mathrm{U} \mathrm{ml}^{-1}$ penicillin and $100 \mu \mathrm{g} \mathrm{ml}^{-1}$ streptomycin. For secondary sphere formation assay, primary spheres were dissociated by using papain and cultured in retinosphere medium.

For retinosphere differentiation assays, retinospheres were plated on poly-Dlysine (Sigma) and Laminin (Invitrogen)-coated dishes (five spheres per $\mathrm{cm}^{2}$ ) and cultured in DMEM/F12-Glutamax medium, 2\% B27 supplement, $20 \mathrm{ng} \mathrm{ml}^{-1}$ human bFGF, $5 \mu \mathrm{ml}^{-1}$ heparin, $0.25 \mu \mathrm{g} \mathrm{ml}^{-1}$ Fungizone, $100 \mathrm{U} \mathrm{ml}^{-1}$ penicillin and $100 \mu \mathrm{g} \mathrm{ml}^{-1}$ streptomycin for 2 days. The cells were further cultured for 10-14 days in retinosphere differentiation medium, containing DMEM/F12-Glutamax medium, $1 \% \mathrm{~N} 2$ supplement, $10 \% \mathrm{FBS}, 0.5 \mu \mathrm{M}$ retinoic acid, $0.1 \mathrm{mM}$ taurine, $10 \mu \mathrm{M}$ DAPT, $0.25 \mu \mathrm{g} \mathrm{ml}^{-1}$ Fungizone, $100 \mathrm{U} \mathrm{ml}^{-1}$ penicillin and $100 \mu \mathrm{g} \mathrm{ml}^{-1}$ streptomycin.

FACS and MACS. For FACS analysis (Fig. 1o,p), cells (from 24 aggregates per each sample, $n=3$ ) were dissociated into single cells using Accumax (Innovative Cell Technologies) and analyzed by using FACSAria with FACSDiva software (Becton Dickinson). For FACS or MACS sorting, dissected tissues were dissociated into single cells by using papain (described above), and then immunostained with an SSEA1-APC antibody (Becton Dickinson; for FACS) or SSEA1-MicroBeads antibody (Miltenyi Biotec; for MACS) at $4{ }^{\circ} \mathrm{C}$ for $15-30 \mathrm{~min}$. For FACS, cells were sorted into $R x::$ Venus-positive/SSEA1-APC ${ }^{+}$fraction and $R x::$ Venus-positive/ SSEA1-APC ${ }^{-}$fractions using FACSAria with the FACSDiva software. For MACS, cells were sorted into SSEA1 ${ }^{+}$or SSEA1 ${ }^{-}$fractions using autoMACS Pro (Miltenyi Biotec) according to manufacturer's instruction.

BrdU and EdU labelling. BrdU and EdU labelling were performed at different time courses as indicated. NR + RPE-containing two-domain aggregates were cultured in NR-differentiation medium in the presence of $\mathrm{BrdU}\left(5 \mu \mathrm{g} \mathrm{ml}^{-1}, 16 \mu \mathrm{M}\right.$; Sigma) for $24 \mathrm{~h}$. Then, aggregates were washed with medium and cultured in the absence of $\mathrm{BrdU}$. For BrdU and EdU double labelling assays, aggregates were further cultured with EdU $(10 \mu \mathrm{M}$; Molecular Probes) in NR-differentiation medium, washed and cultured in the absence of EdU. Aggregates were fixed at $4{ }^{\circ} \mathrm{C}$ in $4 \%$ paraformaldehyde in PBS for $15 \mathrm{~min}$. BrdU was detected by immunostaining. EdU was detected by the Click-it assay according to the manufacturer's protocol.

Immunohistochemistry. Immunohistochemistry was performed as described ${ }^{30}$. Frozen sections were treated with or without heat-based antigen retrieval in Target Retrieval solution $\left(15 \mathrm{~min}\right.$ at $105^{\circ} \mathrm{C}$; DAKO). Primary antibodies used in this study are described as follows: GFP (rabbit/1:500/MBL), (rat/1:500/Nacalai) or (mouse/ 1:1,000/Invitrogen), Pax6 (rabbit/1:200/Covance), Chx10 (goat/1:100/Santa Cruz) or (sheep/1:500/Exalpha), Mitf (mouse/1:500/Exalpha), Sox2 (goat/1:500/Santa Cruz), N-cadherin (Mouse/1:500/BD), phospho-Histone H3 (rabbit/1:500/ Upstate), aPKC (rabbit/1:100/Santa Cruz), Laminin (mouse/BD/1:1,000), Collagen IV (rabbit/ARP/1:1,000), Entactin (rat/Abcam/1:1,000), TuJ1 (mouse/Covance/ 1:500), Blimp1 (rat/1:100/Santa Cruz), Brn3b (goat/1:100/Santa Cruz; C-13), Ptf1a (armenian hamster/1:3; kind gift from Dr Yuichi Ono, KAN Institute), Recoverin (rabbit/1:500/Chemicon), Calbindin (rabbit/1:500/Millipore), Calretinin (rabbit/ 1:1,000/Chemicon), S-opsin (rabbit/1:500/Millipore), Rhodopsin (mouse/1:1,000/ Sigma; RET-P1), NRL (goat/1:500/R\&D), RXR-gamma (rabbit/1:500/Spring Bioscience), Cleaved Caspase 3 (rabbit/1:500/CST), Ki67 (mouse/1:200/BD) or (rabbit/1:200/Novocastra), p27 (C-19; rabbit/1:500/Santa Cruz), p57 (rabbit/1:500/ Sigma), p21 (mouse/1:100/Santa Cruz), BrdU (mouse/1:50/BD), SSEA1 (mouse IgM/1:100/Chemicon), Aqp1 (rabbit/1:500/Millipore), Rdh10 (rabbit/1:200/ Proteintech), Otx1 (mouse/1:1,000/DSHB), Crx (rabbit/1:200/raised in-house), and Zic1 (rabbit/1:20,000/raised in-house). The antiserum against Crx was raised in rabbits against mixed synthetic peptides of $5^{\prime}$-CLEFKDPTGTWKFTYNPMDPLD YKDQSAWKFQIL-3' (Crx 266-299) and 5'-KARPAKRKAGTSPRPSTDVCPDP LGISDSY-3' (Crx 113-143) and was affinity purified. Counter nuclear staining was performed with 4,6-diamidino-2-phenylindole (DAPI; Nacalai). Stained sections were analyzed with an LSM710 confocal microscope (Zeiss).

Live imaging of retinal epithelium. Aggregates with RPE-like thin epithelium on day 24 were embedded in undiluted Matrigel on a $3.5-\mathrm{cm}$ glass-bottom dish (Matsunami) and immersed in either 'RPE-induction medium' (containing DMEM/F12, 1\% N2 supplement, 1\% FBS and $3 \mu \mathrm{M}$ CHIR99021; condition 2) or NR-differentiation medium (described above; condition 3). Live imaging was performed as described ${ }^{30}$ using a specially assembled inverted spinning-disc confocal microscope combined with a full-sized $\mathrm{CO}_{2} / \mathrm{O}_{2}$ incubator. Images were analyzed with the MetaMorph software (Molecular Devices) and Image-J. Similar results were obtained in six aggregates in three independent experiments.

Animals. Pregnant ICR mice were obtained from Japan SLC (Japan). The day of the vaginal plug detection was designated as embryonic day 0.5 (E0.5). All animal experiments were performed in compliance with the Guidelines for Use of Laboratory Animals of RIKEN.
Statistical analysis. Statistical tests were performed with the PRISM software (GraphPad, version 5). Statistical significance was tested with Student's $t$-test (parametric) or Mann-Whitney's test (nonparametric) for two-group comparisons and with analysis of variance followed by post-hoc Tukey's test for multi-group comparisons.

\section{References}

1. Chow, R. L. \& Lang, R. A. Early eye development in vertebrates. Annu. Rev. Cell Dev. Biol. 17, 255-296 (2001).

2. Graw, J. Eye development. Curr. Top. Dev. Biol. 90, 344-385 (2010).

3. Mitashov, V. I. Retinal regeneration in amphibians. Int. J. Dev. Biol. 41, 893-905 (1997).

4. Chiba, C. The retinal pigment epithelium: An important player of retinal disorders and regeneration. Exp. Eye Res. 4835, 00199-1 (2013).

5. Agathocleous, M. \& Harris, W. A. From progenitors to differentiated cells in the vertebrate retina. Annu. Rev. Cell Dev. Biol. 25, 45-69 (2009).

6. Livesey, F. J. \& Cepko, C. L. Vertebrate neural cell-fate determination: lessons from the retina. Nat. Rev. Neurosci. 2, 109-118 (2001).

7. Cayouette, M., Poggi, L. \& Harris, W. A. Lineage in the vertebrate retina Trends Neurosci. 29, 563-570 (2006).

8. Baye, L. M. \& Link, B. A. Interkinetic nuclear migration and the selection of neurogenic cell divisions during vertebrate retinogenesis. J. Neurosci. 27, 10143-10152 (2007).

9. Norden, C., Young, S., Link, B. A. \& Harris, W. A. Actomyosin is the main driver of interkinetic nuclear migration in the retina. Cell 138, 1195-1208 (2009).

10. Bhatia, B., Singhal, S., Jayaram, H., Khaw, P. T. \& Limb, G. A. Adult retinal stem cells revisited. Open Ophthalmol. J. 4, 30-38 (2010).

11. Fischer, A. J., Bosse, J. L. \& El-Hodiri, H. M. The ciliary marginal zone (CMZ) in development and regeneration of the vertebrate eye. Exp. Eye Res. 116, 199-204 (2013).

12. Centanin, L., Hoeckendorf, B. \& Wittbrodt, J. Fate restriction and multipotency in retinal stem cells. Cell Stem Cell 9, 553-562 (2011).

13. Kubo, F., Takeichi, M. \& Nakagawa, S. Wnt2b controls retinal cell differentiation at the ciliary marginal zone. Development 130, 587-598 (2003).

14. Reh, T. A. \& Levine, E. M. Multipotential stem cells and progenitors in the vertebrate retina. J. Neurobiology 36, 206-220 (1998).

15. Trimarchi, J. M., Cho, S. H. \& Cepko, C. L. Identification of genes expressed preferentially in the developing peripheral margin of the optic cup. Dev. Dyn. 238, 2327-2329 (2009).

16. Tropepe, V. et al. Retinal stem cells in the adult mammalian eye. Science $\mathbf{2 8 7}$, 2032-2036 (2000).

17. Coles, B. L. et al. Facile isolation and the characterization of human retinal stem cells. Proc. Natl Acad. Sci. USA 101, 15772-15777 (2004).

18. Martínez-Navarrete, G. C., Angulo, A., Martín-Nieto, J. \& Cuenca, N. Gradual morphogenesis of retinal neurons in the peripheral retinal margin of adult monkeys and humans. J. Comp. Neurol. 511, 557-580 (2008).

19. Bhatia, B., Singhal, S., Lawrence, J. M., Khaw, P. T. \& Limb, G. A. Distribution of Müller stem cells within the neural retina: evidence for the existence of a ciliary margin-like zone in the adult human eye. Exp. Eye Res. 89, 373-382 (2009).

20. Locker, M., Borday, C. \& Perron, M. Stemness or not stemness? Current status and perspectives of adult retinal stem cells. Curr. Stem Cell Res. Ther. 4, $118-130$ (2009).

21. Kawasaki, H. et al. Generation of $\mathrm{TH}^{+}$dopaminergic neurons and $\mathrm{Pax}^{+}$ pigment epithelia from primate ES cells by SDIA. Proc. Natl Acad. Sci. USA 99, 1580-1585 (2002).

22. Ikeda, H. et al. Generation of $\mathrm{Rx}+/ \mathrm{Pax} 6+$ neural retinal precursors from embryonic stem cells. Proc. Natl Acad. Sci. USA 102, 11331-11336 (2005).

23. Lamba, D. A., Karl, M. O., Ware, C. B. \& Reh, T. A. Efficient generation of retinal progenitor cells from human embryonic stem cells. Proc. Natl Acad. Sci. USA 103, 12769-12774 (2006).

24. Osakada, F. et al. Toward generation of rod- and cone-photoreceptors from human and monkey embryonic stem cells under defined culture conditions. Nat. Biotechnol. 26, 215-224 (2008).

25. Idelson, M. et al. Directed differentiation of human embryonic stem cells into functional retinal pigment epithelium cells. Cell Stem Cell 5, 396-408 (2009).

26. Lamba, D. A. et al. Generation, purification and transplantation of photoreceptors derived from human induced pluripotent stem cells. PLoS ONE 5, e8763 (2010).

27. Meyer, J. S. et al. Optic vesicle-like structures derived from human pluripotent stem cells facilitate a customized approach to retinal disease treatment. Stem Cells 29, 1206-1218 (2011).

28. Boucherie, C., Sowden, J. C. \& Ali, R. R. Induced pluripotent stem cell technology for generating photoreceptors. Regen. Med. 6, 469-479 (2011). 
29. Zhu, Y. et al. Three-dimensional neuroepithelial culture from human embryonic stem cells and its use for quantitative conversion to retinal pigment epithelium. PLOS ONE 8, e54552 (2013).

30. Eiraku, M. et al. Self-organizing optic-cup morphogenesis in three-dimensional culture. Nature 472, 51-56 (2011).

31. Ali, R. R. \& Sowden, J. C. DIY eye. Nature 472, 42-43 (2011).

32. Nakano, T. et al. Self-formation of optic cups and storable stratified neural retina from human ES cells. Cell Stem Cell 10, 771-785 (2012).

33. Gonzalez-Cordero, A. et al. Photoreceptor precursors derived from threedimensional embryonic stem cell cultures integrate and mature within adult degenerate retina. Nat. Biotechnol. 31, 741-747 (2013).

34. Mathers, P. H., Grinberg, A., Mahon, K. A. \& Jamrich, M. The Rx homeobox gene is essential for vertebrate eye development. Nature 387, 603-607 (1997).

35. Liu, I. S. et al. Developmental expression of a novel murine homeobox gene (Chx10): evidence for roles in determination of the neuroretina and inner nuclear layer. Neuron 13, 377-393 (1994).

36. Müller, F., Rohrer, H. \& Vogel-Höpker, A. Bone morphogenetic proteins specify the retinal pigment epithelium in the chick embryo. Development 134, 3483-3493 (2007).

37. Fuhrmann, S. Wnt signaling in eye organogenesis. Organogenesis 4, 60-67 (2008).

38. Westenskow, P., Piccolo, S. \& Fuhrmann, S. Beta-catenin controls differentiation of the retinal pigment epithelium in the mouse optic cup by regulating Mitf and Otx2 expression. Development 136, 2505-2510 (2009).

39. Fujimura, N., Taketo, M. M., Mori, M., Korinek, V. \& Kozmik, Z. Spatial and temporal regulation of $\mathrm{Wnt} /$ beta-catenin signaling is essential for development of the retinal pigment epithelium. Dev. Biol. 334, 31-45 (2009).

40. Liu, W., Lagutin, O., Swindell, E., Jamrich, M. \& Oliver, G. Neuroretina specification in mouse embryos requires Six3-mediated suppression of Wnt8b in the anterior neural plate. J. Clin. Invest. 120, 3568-3577 (2010).

41. Park, C. M. \& Hollenberg, M. J. Basic fibroblast growth factor induces retinal regeneration in vivo. Dev. Biol. 134, 201-205 (1989).

42. Guillemot, F. \& Cepko, C. L. Retinal fate and ganglion cell differentiation are potentiated by acidic FGF in an in vitro assay of early retinal development. Development 114, 743-754 (1992).

43. Young, R. W. Cell differentiation in the retina of the mouse. Anat. Rec. 212, 199-205 (1985).

44. O’Brien, K. M., Schulte, D. \& Hendrickson, A. E. Expression of photoreceptorassociated molecules during human fetal eye development. Mol. Vis. 9, 401-409 (2003).

45. Larsen, K. B., Lutterodt, M., Rath, M. F. \& Møller, M. Expression of the homeobox genes PAX6, OTX2, and OTX1 in the early human fetal retina. Int. J. Dev. Neurosci. 27, 485-492 (2009).

46. Nagai, T. et al. The expression of the mouse Zic1, Zic2, and Zic3 gene suggests an essential role for Zic genes in body pattern formation. Dev. Biol. 182, 299-313 (1997).

47. Yamaguchi, Y., Watanabe, T., Hirakata, A. \& Hida, T. Localization and ontogeny of aquaporin- 1 and -4 expression in iris and ciliary epithelial cells in rats. Cell Tissue Res. 325, 101-109 (2006).

48. Koso, H. et al. CD138/syndecan-1 and SSEA-1 mark distinct populations of developing ciliary epithelium that are regulated differentially by Wnt signal. Stem Cells 26, 3162-3171 (2008).

49. Kubo, F. \& Nakagawa, S. Hairyl acts as a node downstream of Wnt signaling to maintain retinal stem cell-like progenitor cells in the chick ciliary marginal zone. Development 136, 1823-1833 (2009).

50. Dyer, M. A. \& Cepko, C. L. p57(Kip2) regulates progenitor cell proliferation and amacrine interneuron development in the mouse retina. Development 127, 3593-3605 (2000).

51. Levine, E. M., Close, J., Fero, M., Ostrovsky, A. \& Reh, T. A. p27(Kip1) regulates cell cycle withdrawal of late multipotent progenitor cells in the mammalian retina. Dev. Biol. 219, 299-314 (2000).

52. Jadhav, A. P., Cho, S. H. \& Cepko, C. L. Notch activity permits retinal cells to progress through multiple progenitor states and acquire a stem cell property. Proc. Natl Acad. Sci. USA 103, 18998-19003 (2006).

53. Clarke, L., Ballios, B. G. \& van der Kooy, D. Generation and clonal isolation of retinal stem cells from human embryonic stem cells. Eur. J. Neurosci. 36, 1951-1959 (2012)
54. Rowan, S., Chen, C. M., Young, T. L., Fisher, D. E. \& Cepko, C. L. Transdifferentiation of the retina into pigmented cells in ocular retardation mice defines a new function of the homeodomain gene Chx10. Development 131, 5139-5152 (2004).

55. Salero, E. et al. Adult human RPE can be activated into a multipotent stem cell that produces mesenchymal derivatives. Cell Stem Cell 10, 88-95 (2012).

56. Sasai, Y. Cytosystems dynamics in self-organization of tissue architecture. Nature 493, 318-326 (2013).

57. Yasumoto, K. et al. Microphthalmia-associated transcription factor interacts with LEF-1, a mediator of Wnt signaling. EMBO J. 21, 2703-2714 (2002).

58. Liu, H., Mohamed, O., Dufort, D. \& Wallace, V. A. Characterization of Wnt signaling components and activation of the Wnt canonical pathway in the murine retina. Dev. Dyn. 227, 323-334 (2003).

59. Diep, D. B., Hoen, N., Backman, M., Machon, O. \& Krauss, S. Characterisation of the Wnt antagonists and their response to conditionally activated Wnt signalling in the developing mouse forebrain. Brain Res. Dev. Brain Res. 153, 261-270 (2004).

60. Hendrickson, A. \& Provis, J. in Retinal Development (eds Sernagor, E., Eglen, S., Harris, B. \& Wong, R.) (Cambridge Univ. Press, 2006).

61. Cicero, S. A. et al. Cells previously identified as retinal stem cells are pigmented ciliary epithelial cells. Proc. Natl Acad. Sci. USA 106, 6685-6690 (2009)

62. Lawrence, J. M. et al. MIO-M1 cells and similar muller glial cell lines derived from adult human retina exhibit neural stem cell characteristics. Stem Cells 25, 2033-2043 (2007).

63. Furuta, Y., Piston, D. W. \& Hogan, B. L. Bone morphogenetic proteins (BMPs) as regulators of dorsal forebrain development. Development 124, 2203-2212 (1997).

64. Hanashima, C., Li, S. C., Shen, L., Lai, E. \& Fishell, G. Foxg1 suppresses early cortical cell fate. Science 303, 56-59 (2004).

65. Rubenstein, J. L., Shimamura, K., Martinez, S. \& Puelles, L. Regionalization of the prosencephalic neural plate. Annu. Rev. Neurosci. 21, 445-477 (1998).

66. Dale, K. et al. Differential patterning of ventral midline cells by axial mesoderm is regulated by BMP7 and chordin. Development 126, 397-408 (1999).

67. Ohyama, K., Das, R. \& Placzek, M. Temporal progression of hypothalamic patterning by a dual action of BMP. Development 135, 3325-3331 (2008).

68. Watanabe, K. et al. A ROCK inhibitor permits survival of dissociated human embryonic stem cells. Nat. Biotechnol. 25, 681-686 (2007).

69. Wataya, T. et al. Minimization of exogenous signals in ES cell culture induces rostral hypothalamic differentiation. Proc. Natl Acad. Sci. USA 105, 11796-11801 (2008).

\section{Acknowledgements}

This paper is dedicated to Dr Yoshiki Sasai, an incredible mentor who suddenly passed away on 5 August 2014. We are grateful to Nick Love, Keiko Muguruma, Momoko Watanabe, Taisuke Kadoshima and Daiki Nukaya for invaluable comments, and Rieko Yakura for technical support. This work was supported by grants-in-aid from MEXT (Y.S., M.E.), the S-Innovation from JST (K.S.) and the Research Center Network for Realization of Regenerative Medicine from JST (Y.S.).

\section{Author contributions}

A.K., C.O. and T.N. performed the experiments; A.K. and Y.S. wrote the manuscript; A.K., K.S., M.E. and Y.S. designed the project.

\section{Additional information}

Supplementary Information accompanies this paper at http://www.nature.com/ naturecommunications

Competing financial interests: A.K., T.N. and K.S. are employed by Sumitomo Chemical Co., Ltd (SCC). The authors are co-inventors on patent applications filed by RIKEN and SCC. The authors declare no other competing financial interests.

Reprints and permission information is available online at http://npg.nature.com/ reprintsandpermissions/

How to cite this article: Kuwahara, A. et al. Generation of a ciliary margin-like stem cell niche from self-organizing human retinal tissue. Nat. Commun. 6:6286 doi: $10.1038 /$ ncomms7286 (2015). 